Open Access

\title{
Probiotics for prevention of necrotizing enterocolitis in preterm infants: systematic review and meta-analysis
}

Arianna Aceti ${ }^{* *}$, Davide Gori ${ }^{2}$, Giovanni Barone ${ }^{3}$, Maria Luisa Callegari ${ }^{4}$, Antonio Di Mauro ${ }^{5}$, Maria Pia Fantini ${ }^{2}$, Flavia Indrio ${ }^{5}$, Luca Maggio ${ }^{3}$, Fabio Meneghin ${ }^{6}$, Lorenzo Morelli ${ }^{4}$, Gianvincenzo Zuccotti ${ }^{6}$, Luigi Corvaglia ${ }^{1}$ and on behalf of the Italian Society of Neonatology

\begin{abstract}
Necrotizing enterocolitis (NEC) affects predominantly preterm infants, who have specific risk factors leading to intestinal dysbiosis. Manipulations of gut microbiota through probiotics have the potential to prevent NEC.

The aim of this systematic review and meta-analysis was to evaluate the effect of probiotics for NEC prevention in preterm infants, with a focus on specific strains, microbiological strength of currently available studies, and high-risk populations.

PubMed and the Cochrane Library were searched for trials published within 4th February 2015. Randomized-controlled trials reporting on NEC and involving preterm infants who were given probiotics in the first month of life were included in the systematic review.

Twenty-six studies were suitable for inclusion in the meta-analysis.

Data about study design, population, intervention and outcome were extracted and summarized independently by two observers. Study quality and quality of evidence were also evaluated.

Fixed-effects models were used and random-effects models where significant heterogeneity was present. Subgroup analyses were performed to explore sources of heterogeneity among studies. Results were expresses as risk ratio (RR) with $95 \%$ confidence interval $(\mathrm{Cl})$.

The main outcome was incidence of NEC stage $\geq 2$ according to Bell's criteria.

Probiotics prevented NEC in preterm infants (RR 0.47 [95 \% Cl 0.36-0.60], $p<0.00001$ ). Strain-specific sub-meta-analyses showed a significant effect for Bifidobacteria (RR 0.24 [95 \% Cl 0.10-0.54], $p=0.0006$ ) and for probiotic mixtures (RR 0.39 [95 \% Cl 0.27-0.56], $p<0.00001$ ). Probiotics prevented NEC in very-low-birth-weight infants (RR 0.48 [95 \% Cl 0.37-0.62], $p<0.00001$ ); there were insufficient data for extremely-low-birth-weight infants. The majority of studies presented severe or moderate microbiological flaws.

Probiotics had an overall preventive effect on NEC in preterm infants. However, there are still insufficient data on the specific probiotic strain to be used and on the effect of probiotics in high-risk populations such as extremely-low-birthweight infants, before a widespread use of these products can be recommended.
\end{abstract}

Keywords: Probiotics, Newborn, Necrotizing enterocolitis, Meta-analysis

\footnotetext{
*Correspondence: arianna.aceti2@unibo.it

${ }^{1}$ Neonatal Intensive Care Unit, Department of Medical and Surgical Sciences (DIMEC), University of Bologna, S.Orsola-Malpighi Hospital, Bologna, Italy

Full list of author information is available at the end of the article
}

\section{Ciomed Central}

(c) 2015 Aceti et al. Open Access This article is distributed under the terms of the Creative Commons Attribution 4.0 International License (http://creativecommons.org/licenses/by/4.0/, which permits unrestricted use, distribution, and reproduction in any medium, provided you give appropriate credit to the original author(s) and the source, provide a link to the Creative Commons license, and indicate if changes were made. The Creative Commons Public Domain Dedication waiver (http://creativecommons.org/publicdomain/zero/1.0/) applies to the data made available in this article, unless otherwise stated. 


\section{Background}

Necrotizing enterocolitis (NEC), which is one of the most devastating neonatal diseases, has become a priority for research [1]. Despite great advances in neonatal care, the morbidity, mortality and health-care costs directly related to the disease are substantial: during hospital stay, the economic burden of NEC in the United States has been estimated as high as several billions USD per year, which is approximately $20 \%$ of the costs for Neonatal Intensive Care Units in the country; furthermore, this estimate is likely to be much higher when the costs of long-term care of survivors are taken into account [2].

NEC is a multifactorial disease: prematurity is a wellrecognized risk factor, and approximately $90 \%$ of the infants who develop NEC are born preterm [3]. This is probably due to specific comorbidities of prematurity, such as immunodeficiency, use of broad-spectrum antimicrobials, delayed enteral feeding and low availability of human milk.

Recently, research has focused on the role of gut microbiota and its manipulations, such as the use of probiotics, on disease and health status. Probiotics are live-microorganisms which, when ingested in adequate amounts, confer a health-benefit to the host through an interaction with gut microbiota [4]. The intestinal microbiota undergoes dynamic changes during childhood. Gut colonization in preterm infants occurs differently than in healthy term newborns [5], and preterm infants frequently have delayed and aberrant acquisition of the "normal" digestive flora. Recent studies performed in preterm foetuses and infants demonstrated that amniotic fluid and meconium are not sterile, suggesting an intrauterine origin of gut microbiota [6, 7]; after birth, the preterm infant's immature intestine is exposed to an unique environment and to several iatrogenic manipulations, including the use of broad-spectrum antibiotics. The subsequent intestinal dysbiosis is recognized as a risk factor for NEC: actually, it has been shown that preterm infants with NEC have reduced bacterial gut diversity and different bacterial strains compared to healthy controls [8]. In this perspective, provision of probiotics to preterm infants has the potential to "normalize" the abnormal colonization pattern, thus preventing the occurrence of the disease [9].

The use of probiotics for the prevention of NEC in preterm infants has been extensively investigated in many randomized-controlled trials, whose results have been summarized in several systematic-reviews and metaanalyses [10, 11]. The authors of these meta-analyses, which show that probiotics reduce NEC and mortality in preterm infants, strongly encourage a change in practice, promoting a widespread use of probiotics in this population [11], and also claim that withholding probiotics from high-risk neonates would be almost unethical [10].
However, the position of the American Academy of Paediatrics is more cautious, highlighting the need for more studies to address unanswered questions on the amount and specificity of which probiotic or mixture of probiotics should be used [12]. In addition, a recent systematic review, which analyzed the level of evidence of randomized-controlled trials on probiotics in preterm infants, concluded that there is still insufficient evidence to recommend routine probiotics use, but also that present data are encouraging and justify further research on specific probiotic products [13].

Actually, the beneficial effects of probiotics appear to be strain-specific, and pooling data from studies using different strains can result in misleading conclusions [14]. Furthermore, currently available studies often lack specificity in reporting correct identification of probiotic strain [15], dosage regimen and duration, and gut colonization, which are all fundamental to assess the ability of a probiotic to confer a health benefit to the host [16].

The aim of this meta-analysis is thus to evaluate in detail the effect of probiotics for the prevention of NEC in preterm infants, with a focus on specific strains, on microbiological strength of currently available studies, and on high-risk populations.

\section{Methods}

\section{Literature search}

The study protocol was designed jointly by the members of the Task Force on Probiotics of the Italian Society of Neonatology.

A systematic review of published studies reporting the use of probiotics for the prevention of NEC in preterm infants was performed, following PRISMA guidelines [17].

Criteria for inclusion in the meta-analysis were the following: randomized and quasi-randomized controlled trials involving preterm infants (gestational age $<37$ weeks) and reporting on NEC (any stage, according to modified Bell staging criteria $[18,19])$; enteral administration of any probiotic starting within one month of age, compared to placebo or no treatment. Being the search strategy focused specifically on NEC, data on different outcomes, such as sepsis or mortality, which were reported in the studies retrieved by the literature search, were not evaluated by meta-analysis.

A search was conducted in PubMed (http://www. ncbi.nlm.nih.gov/pubmed/) for studies published before 4th February 2015, using the search string reported in Fig. 1. This string was built up combining all the terms related to NEC and probiotics, using PubMed MeSH terms and free-text words and their combinations through the most proper Boolean operators, in order to be as comprehensive as possible. Similar criteria were used for searching the Cochrane Library. The review was limited to studies written in English and involving human subjects. 


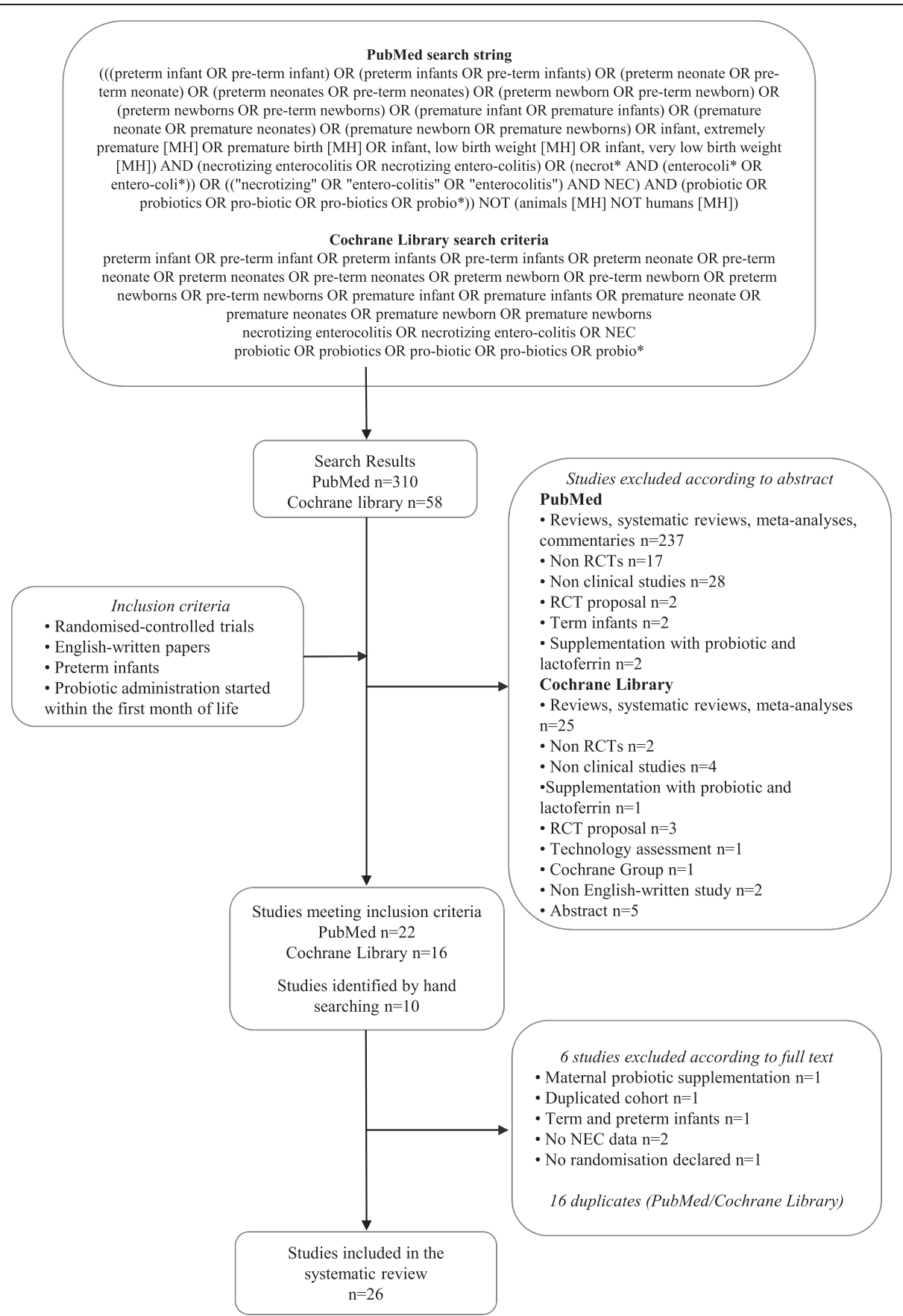

Fig. 1 Flow chart showing the search strategy and search results. The relevant number of papers at each point is given

The search was conducted by AA and LC: relevant studies were identified from the abstract, and reference lists of papers retrieved were searched for additional studies. "Snowballing" technique was also used [20].

\section{Data extraction and meta-analysis}

Study details, including study population, characteristics of the intervention, use of placebo, and outcome, were assessed independently by AA and LC, and checked by DG. Study quality was evaluated independently using the risk of bias tool as proposed by the Cochrane collaboration (Chapter 8 of the Cochrane Handbook of Systematic Reviews) [21]. In addition, an assessment of the body of evidence using the GRADE working group approach was used in order to grade the quality of evidence. The evaluation was carried out following the Chapter 12 of the Cochrane Handbook [21] and classifying the evidence as high, 
Table 1 Studies included in the systematic review and meta-analysis

\begin{tabular}{|c|c|c|c|c|c|}
\hline \multirow[t]{5}{*}{ Author, year } & \multirow{5}{*}{$\begin{array}{l}\text { Study } \\
\text { details }\end{array}$} & \multirow[t]{5}{*}{ Study population } & Intervention & \multirow[t]{5}{*}{ Type of milk } & \multirow[t]{5}{*}{ Placebo } \\
\hline & & & - Strain & & \\
\hline & & & - Dose (D) & & \\
\hline & & & - Start of treatment $(S)$ & & \\
\hline & & & - End of treatment (E) & & \\
\hline \multirow[t]{5}{*}{ Al-Hosni, 2012 [33] } & $P$ & \multirow{5}{*}{$\begin{array}{l}\text { Preterm infants with BW } 501-1000 \mathrm{~g} \text {, appropriate for } \\
\text { gestational age, and } \leq 14 \text { days of age at time of } \\
\text { feeding initiation }\end{array}$} & Lactobacillus rhamnosus GG LGG & \multirow[t]{5}{*}{ Non specified } & \multirow[t]{5}{*}{ Extra milk } \\
\hline & DB & & Bifidobacterium infantis & & \\
\hline & R & & D: $0.5 \times 10^{9} \mathrm{CFU}$ each probiotic, OD & & \\
\hline & C & & S: first enteral feeding & & \\
\hline & Multic. & & E: discharge or until $34 \mathrm{w}$ postmenstrual age & & \\
\hline \multirow[t]{6}{*}{ Bin-Nun, 2005 [40] } & $P$ & \multirow{6}{*}{$\begin{array}{l}\text { Preterm infants with BW }<1500 \mathrm{~g} \text {, who began enteral } \\
\text { feeding on a weekday }\end{array}$} & Bifidobacterium infantis & \multirow[t]{6}{*}{ OMM, PFM } & \multirow[t]{6}{*}{$\mathrm{HM}$ or $\mathrm{FM}$} \\
\hline & B & & Streptococcus thermophilus & & \\
\hline & $\mathrm{R}$ & & Bifidobacterium bifidus & & \\
\hline & \multirow[t]{3}{*}{ C } & & D: $0.35 \times 10^{9}$ CFU each probiotic, OD & & \\
\hline & & & S: start of enteral feeding & & \\
\hline & & & E: $36 \mathrm{w}$ postconceptual age & & \\
\hline \multirow[t]{5}{*}{ Braga, 2011 [35] } & $P$ & \multirow[t]{5}{*}{ Inborn infants with BW 750-1499 g } & Lactobacillus casei & \multirow[t]{5}{*}{$\mathrm{HM}$} & \multirow[t]{5}{*}{ Extra HM } \\
\hline & DB & & Bifidobacterium breve & & \\
\hline & $\mathrm{R}$ & & D: $3.5 \times 10^{7} \mathrm{CFU}$ to $3.5 \times 10^{9} \mathrm{CFU}$ OD & & \\
\hline & \multirow[t]{2}{*}{ C } & & S: day 2 & & \\
\hline & & & $\begin{array}{l}\text { E: day 30, NEC diagnosis, discharge, death, whichever } \\
\text { occurred first }\end{array}$ & & \\
\hline \multirow[t]{4}{*}{ Costalos, 2003 [49] } & $P$ & GA 28-32 w & Saccharomyces boulardii & \multirow[t]{4}{*}{ PFM } & \multirow[t]{4}{*}{ MDX } \\
\hline & $\mathrm{R}$ & No major Gl problem & $\mathrm{D}: 1 \times 10^{9} \mathrm{CFU} B \mathrm{BD}$ & & \\
\hline & \multirow[t]{2}{*}{$C$} & Not receiving antibiotics & S: non-specified & & \\
\hline & & Not receiving breast milk & Median duration of probiotic supplementation: 30 days & & \\
\hline \multirow[t]{5}{*}{ Dani, 2002 [42] } & P & \multirow[t]{5}{*}{ Infants with GA $<33$ w or BW $<1500 \mathrm{~g}$} & Lactobacillus rhamnosus GG & \multirow[t]{5}{*}{ OMM, DM or FM } & \multirow[t]{5}{*}{ MDX } \\
\hline & DB & & D: $6 \times 10^{9}$ CFU OD & & \\
\hline & $\mathrm{R}$ & & S: first feed & & \\
\hline & C & & E: discharge & & \\
\hline & Multic. & & & & \\
\hline \multirow[t]{3}{*}{ Demirel, 2013 [28] } & $P$ & \multirow{3}{*}{$\begin{array}{l}\text { Preterm infants with } G A \leq 32 \mathrm{w} \text { and } \mathrm{BW} \leq 1500 \mathrm{~g} \text {, who } \\
\text { survived to feed enterally }\end{array}$} & Saccharomyces boulardii & \multirow[t]{3}{*}{$\mathrm{HM}, \mathrm{FM}$} & None \\
\hline & B & & D: $5 \times 10^{9}$ CFU OD & & \\
\hline & $\mathrm{R}$ & & S: first feed & & \\
\hline
\end{tabular}


Table 1 Studies included in the systematic review and meta-analysis (Continued)

\begin{tabular}{|c|c|c|c|c|c|}
\hline & C & & E: discharge & & \\
\hline \multirow[t]{5}{*}{ Dilli, 2015 [44] } & P & \multirow{5}{*}{$\begin{array}{l}\text { Preterm infants with GA }<32 \text { weeks and BW }<1500 \mathrm{~g} \text {, } \\
\text { born at or transferred to the NICU within the first week } \\
\text { of life and fed enterally before inclusion }\end{array}$} & Bifidobacterium & \multirow[t]{5}{*}{$\mathrm{HM}, \mathrm{FM}$} & \multirow[t]{5}{*}{ MDX powder } \\
\hline & $\mathrm{DB}$ & & Lactis & & \\
\hline & $\mathrm{R}$ & & D: $5 \times 10^{9} \mathrm{CFU}$ & & \\
\hline & C & & S: beyond d7 after birth & & \\
\hline & Multic. & & E: death or discharge (max 8 weeks) & & \\
\hline \multirow{9}{*}{$\begin{array}{l}\text { Fernández-Carrocera, } \\
2013 \text { [32] }\end{array}$} & P & Preterm infants with $\mathrm{BW}<1500 \mathrm{~g}$ & Lactobacillus acidophilus 1 CFU/g & \multirow[t]{9}{*}{ OMM, PFM } & \multirow[t]{9}{*}{ None } \\
\hline & DB & \multirow[t]{8}{*}{ Infants with NEC IA and IB were excluded } & Lactobacillus rhamnosus $4.4 \times 10^{8} \mathrm{CFU} / \mathrm{g}$ & & \\
\hline & R & & Lactobacillus casei $1 \times 10^{9} \mathrm{CFU} / \mathrm{g}$ & & \\
\hline & \multirow[t]{6}{*}{ C } & & Lactobacillus plantarum $1.76 \times 10^{8} \mathrm{CFU} / \mathrm{g}$ & & \\
\hline & & & Bifidobacterium infantis $2.76 \times 10^{7} \mathrm{CFU} / \mathrm{g}$ & & \\
\hline & & & Streptococcus thermophilus $6.6 \times 10^{5} \mathrm{CFU} / \mathrm{g}$ & & \\
\hline & & & Total D: $1 \mathrm{~g}$ powder OD & & \\
\hline & & & S: start of enteral feeding & & \\
\hline & & & E: non-specified & & \\
\hline \multirow[t]{6}{*}{ Jacobs, 2013 [26] } & P & \multirow[t]{6}{*}{ Preterm infants with GA $<32 \mathrm{w}$ and $\mathrm{BW}<1500 \mathrm{~g}$} & Bifidobacterium infantis BB-02 $300 \mathrm{CFU} \times 10^{6}$ & \multirow[t]{6}{*}{$H M, F M$} & \multirow[t]{6}{*}{ MDX powder } \\
\hline & DB & & Streptococcus thermophilus Th-4 $350 \mathrm{CFU} \times 10^{6}$ & & \\
\hline & $\mathrm{R}$ & & Bifidobacterium lactis BB-12 $350 \mathrm{CFU} \times 10^{6}$ & & \\
\hline & C & & Total D: $1 \times 10^{9} \mathrm{CFU} \times 1.5 \mathrm{~g}$ maltodextrin powder $\mathrm{OD}$ & & \\
\hline & \multirow[t]{2}{*}{ Multic. } & & S: enteral feed $\geq 1 \mathrm{ml}$ every $4 \mathrm{~h}$ & & \\
\hline & & & E: discharge or term corrected age & & \\
\hline \multirow[t]{4}{*}{ Kitajima, 1997 [52] } & P & \multirow[t]{4}{*}{ Preterm infants with $\mathrm{BW}<1500 \mathrm{~g}$} & Bifidobacterium breve YIT4010 & \multirow{4}{*}{$\begin{array}{l}\text { OMM, FM after full enteral feeding } \\
\text { had been reached }\end{array}$} & \multirow[t]{4}{*}{ Distilled water } \\
\hline & $\mathrm{R}$ & & D: $0.5 \times 10^{9} \mathrm{CFU}$ OD & & \\
\hline & C & & S: within $24 \mathrm{~h}$ of life & & \\
\hline & & & Duration of probiotic supplementation: 28 days & & \\
\hline \multirow[t]{5}{*}{ Lin, 2005} & P & \multirow{5}{*}{$\begin{array}{l}\text { Infants with BW }<1500 \mathrm{~g} \text {, who started to feed enterally } \\
\text { and survived beyond day } 7\end{array}$} & Lactobacillus acidophilus & \multirow[t]{5}{*}{ OMM, DM } & \multirow[t]{5}{*}{ None } \\
\hline & M & & Bifidobacterium infantis & & \\
\hline & $\mathrm{R}$ & & D: $\geq 10^{6} \mathrm{CFU}$ each probiotic ( $\left.=125 \mathrm{mg} / \mathrm{kg}\right), \mathrm{BD}$ & & \\
\hline & C & & S: start of enteral feeding & & \\
\hline & & & E: discharge & & \\
\hline \multirow[t]{3}{*}{ Lin, 2008} & P & \multirow{3}{*}{$\begin{array}{l}\text { Preterm infants with } \mathrm{GA}<34 \mathrm{w} \text { and } \mathrm{BW} \leq 1500 \mathrm{~g} \text {, who } \\
\text { survived to feed enterally }\end{array}$} & Lactobacillus acidophilus NCDO 1746 & \multirow[t]{3}{*}{$\mathrm{HM}, \mathrm{FM}$} & \multirow[t]{3}{*}{ None } \\
\hline & B & & Bifidobacterium bifidum NCDO 1453 & & \\
\hline & $\mathrm{R}$ & & D: $1 \times 10^{9} \mathrm{CFU}$ each probiotic $(=125 \mathrm{mg} / \mathrm{kg}) \mathrm{BD}$ & & \\
\hline
\end{tabular}


Table 1 Studies included in the systematic review and meta-analysis (Continued)

\begin{tabular}{|c|c|c|c|c|c|}
\hline & C & & S: day 2 of age & & \\
\hline & Multic. & & Duration: 6 weeks & & \\
\hline \multirow[t]{4}{*}{ Manzoni, 2006 [37] } & $P$ & \multirow{4}{*}{$\begin{array}{l}\text { Infants with } \mathrm{BW}<1500 \mathrm{~g}, \geq 3 \text { days of life, who started } \\
\text { enteral feeding with } \mathrm{HM}\end{array}$} & Lactobacillus rhamnosus LGG & \multirow[t]{4}{*}{ OMM, DM } & \multirow[t]{4}{*}{ None } \\
\hline & $\mathrm{DB}$ & & D: $6 \times 10^{9} \mathrm{CFU} /$ day & & \\
\hline & $\mathrm{R}$ & & S: day 3 of life & & \\
\hline & C & & E: end of the 6th week or discharge & & \\
\hline \multirow[t]{4}{*}{ Mihatsch, 2010 [43] } & $P$ & \multirow[t]{4}{*}{ Preterm infants with $G A<30 \mathrm{w}$ and $\mathrm{BW} \leq 1500 \mathrm{~g}$} & Bifidobacterium lactis BB12 & \multirow[t]{4}{*}{ HM, PFM } & \multirow{4}{*}{$\begin{array}{l}\text { Indistinguishable } \\
\text { powder }\end{array}$} \\
\hline & $\mathrm{R}$ & & D: $2 \times 10^{9} \mathrm{CFU} / \mathrm{kg} 6$ times a day & & \\
\hline & C & & S: start of enteral feeding & & \\
\hline & & & E: non-specified & & \\
\hline \multirow[t]{4}{*}{ Mohan, 2006 [53] } & P & \multirow[t]{4}{*}{ Preterm infants $(G A<37$ w) } & Bifidobacterium lactis BB12 & \multirow{4}{*}{ FM } & \multirow[t]{4}{*}{ Not stated } \\
\hline & $\mathrm{DB}$ & & $\begin{array}{l}\text { D: } 1.6 \times 10^{9} \text { CFU on day } 1 \text { to } 3 \text {, and } 4.8 \times 10^{9} \text { CFU from } \\
\text { day } 4 \text { onwards }\end{array}$ & & \\
\hline & $\mathrm{R}$ & & S: first day of life & & \\
\hline & C & & Duration: 21 days & & \\
\hline \multirow[t]{4}{*}{ Oncel, 2013 [25] } & P & \multirow{4}{*}{$\begin{array}{l}\text { Preterm infants with GA } \leq 32 \mathrm{w} \text { and } \mathrm{BW} \leq 1500 \mathrm{~g} \text {, who } \\
\text { survived to feed enterally }\end{array}$} & Lactobacillus reuteri DSM 17938 & \multirow[t]{4}{*}{ HM, FM } & \multirow[t]{4}{*}{ Oil base } \\
\hline & DB & & D: $1 \times 10^{8}$ CFU OD & & \\
\hline & $\mathrm{R}$ & & S: first feed & & \\
\hline & C & & E: death or discharge & & \\
\hline \multirow[t]{4}{*}{ Patole, 2014 [45] } & $P$ & \multirow[t]{4}{*}{ Preterm infants with $\mathrm{GA}<33 \mathrm{w}$ and $\mathrm{BW}<1500 \mathrm{~g}$} & Bifidobacterium breve M16-V & \multirow[t]{4}{*}{$\mathrm{HM}, \mathrm{FM}$} & \multirow[t]{4}{*}{ Dextrin } \\
\hline & DB & & $\begin{array}{l}\text { D: } 3 \times 10^{9} \mathrm{CFU} \text { OD }(1.5 \times 109 \mathrm{CFU} \text { OD for newborns } \\
\leq 27 \mathrm{w} \text { until they reached } 50 \mathrm{ml} / \mathrm{kg} / \text { day enteral feeds })\end{array}$ & & \\
\hline & $\mathrm{R}$ & & S: start of enteral feed & & \\
\hline & C & & E: corrected age of $37 \mathrm{w}$ & & \\
\hline \multirow[t]{5}{*}{ Rojas, 2012 [30] } & P & \multirow{5}{*}{$\begin{array}{l}\text { Preterm infants with BW } \leq 2000 \mathrm{~g} \text {, hemodynamically } \\
\text { stable, } \leq 48 \mathrm{~h} \text { of age (regardless start of enteral feeding) }\end{array}$} & Lactobacillus reuteri DSM 17938 & \multirow[t]{5}{*}{ HM, FM } & \multirow[t]{5}{*}{ Oil base } \\
\hline & DB & & D: $1 \times 10^{8}$ CFU OD & & \\
\hline & $\mathrm{R}$ & & S: age $\leq 48 \mathrm{~h}$ & & \\
\hline & C & & E: death or discharge & & \\
\hline & Multic. & & & & \\
\hline \multirow[t]{5}{*}{ Rougé, 2009 [50] } & P & \multirow{5}{*}{$\begin{array}{l}\text { Preterm infants with } \mathrm{GA}<32 \mathrm{w} \text { and } \mathrm{BW}<1500 \mathrm{~g} \text {, } \\
\leq 2 \mathrm{w} \text { of age, without any disease other than those } \\
\text { linked to prematurity, who started enteral feeding } \\
\text { before inclusion }\end{array}$} & Bifidobacterium longum BB536 & \multirow[t]{5}{*}{ OMM, DM or PFM } & \multirow[t]{5}{*}{ MDX } \\
\hline & $\mathrm{DB}$ & & Lactobacillus rhamnosus GG BB536-LGG & & \\
\hline & $\mathrm{R}$ & & Total D: $1 \times 10^{8}$ CFU/day & & \\
\hline & C & & S: start of enteral feeding & & \\
\hline & Bic. & & E: discharge & & \\
\hline
\end{tabular}


Table 1 Studies included in the systematic review and meta-analysis (Continued)

\begin{tabular}{|c|c|c|c|c|c|}
\hline \multirow[t]{7}{*}{ Roy, 2014 [58] } & P & \multirow{7}{*}{$\begin{array}{l}\text { Preterm infants }(G A<37 w) \text { and } B W<2500 \mathrm{~g} \text {, with stable } \\
\text { enteral feeding within } 72 \mathrm{~h} \text { of birth }\end{array}$} & Lactobacillus acidophilus $1.25 \times 10^{9} \mathrm{CFU} \times 1 \mathrm{~g}$ & \multirow[t]{7}{*}{$\mathrm{HM}$} & \multirow[t]{7}{*}{ Sterile water } \\
\hline & $\mathrm{DB}$ & & B. longum $0.125 \times 10^{9} \mathrm{CFU} \times 1 \mathrm{~g}$ & & \\
\hline & $\mathrm{R}$ & & B. bifidum $0.125 \times 10^{9} \mathrm{CFU} \times 1 \mathrm{~g}$ & & \\
\hline & \multirow[t]{4}{*}{ C } & & B. lactis $1 \times 10^{9} \mathrm{CFU} \times 1 \mathrm{~g}$ & & \\
\hline & & & D: half a $1 \mathrm{~g}$ sachet & & \\
\hline & & & S: from $72 \mathrm{~h}$ of life & & \\
\hline & & & E: after $6 w$ or at discharge & & \\
\hline \multirow[t]{6}{*}{ Saengtawesin, 2014 [48] } & $\mathrm{P}$ & \multirow[t]{6}{*}{ Preterm infants with $\mathrm{GA} \leq 34 \mathrm{w}$ and $\mathrm{BW} \leq 1500 \mathrm{~g}$} & Lactobacillus acidophilus $1 \times 10^{9} \mathrm{CFU}$ & \multirow[t]{6}{*}{ HM, PFM } & \multirow[t]{6}{*}{ None } \\
\hline & $\mathrm{R}$ & & Bifidobacterium & & \\
\hline & \multirow[t]{4}{*}{ C } & & bifidum $1 \times 10^{9} \mathrm{CFU}$ & & \\
\hline & & & D: 125 mg/kg BD & & \\
\hline & & & S: start of feeding & & \\
\hline & & & E: 6 w of age or discharge. & & \\
\hline \multirow[t]{7}{*}{ Samanta, 2009} & $P$ & \multirow{7}{*}{$\begin{array}{l}\text { Preterm infants with } G A<32 \mathrm{w} \text { and } \mathrm{BW}<1500 \mathrm{~g} \text {, who } \\
\text { started enteral feeding and survived beyond } 48 \mathrm{~h} \text { of age }\end{array}$} & Bifidobacterium infantis & \multirow[t]{7}{*}{$\mathrm{HM}$} & \multirow[t]{7}{*}{ None } \\
\hline & DB & & Bifidobacterium bifidum & & \\
\hline & $\mathrm{R}$ & & Bifidobacterium longum & & \\
\hline & \multirow[t]{4}{*}{ C } & & Lactobacillus acidophilus & & \\
\hline & & & D: $2.5 \times 10^{9}$ CFU each probiotic, BD & & \\
\hline & & & S: start of enteral feeding & & \\
\hline & & & E: discharge & & \\
\hline \multirow[t]{4}{*}{ Sari, 2011 [34] } & $P$ & \multirow{4}{*}{$\begin{array}{l}\text { Preterm infants with } \mathrm{GA}<32 \mathrm{w} \text { or } \mathrm{BW}<1500 \mathrm{~g} \text {, who } \\
\text { survived to feed enterally }\end{array}$} & Lactobacillus sporogenes & \multirow[t]{4}{*}{$\mathrm{HM}, \mathrm{FM}$} & \multirow[t]{4}{*}{ None } \\
\hline & B & & D: $0.35 \times 10^{9} \mathrm{CFU}$ OD & & \\
\hline & $\mathrm{R}$ & & S: first feed & & \\
\hline & C & & E: discharge & & \\
\hline \multirow[t]{4}{*}{ Serce, 2013 [27] } & P & \multirow{4}{*}{$\begin{array}{l}\text { Preterm infants with } G A \leq 32 \mathrm{w} \text { and } \mathrm{BW} \leq 1500 \mathrm{~g} \text {, who } \\
\text { survived to feed enterally }\end{array}$} & Saccharomyces boulardii & \multirow[t]{4}{*}{$\mathrm{HM}, \mathrm{FM}$} & \multirow[t]{4}{*}{ Distilled water } \\
\hline & M & & $\mathrm{D}: 0.5 \times 10^{9} \mathrm{CFU} / \mathrm{kg} \mathrm{BD}$ & & \\
\hline & $\mathrm{R}$ & & S: non-specified & & \\
\hline & C & & E: non-specified & & \\
\hline \multirow[t]{4}{*}{ Stratiki, 2007 [39] } & $P$ & \multirow{4}{*}{$\begin{array}{l}\text { Preterm infants with GA } 27-32 \text { w, formula-fed, without } \\
\text { major congenital anomalies }\end{array}$} & Bifidobacterium lactis & \multirow[t]{4}{*}{ FM } & \multirow[t]{4}{*}{ None } \\
\hline & B & & D: $2 \times 10^{7} \mathrm{CFU} / \mathrm{g}$ of milk powder & & \\
\hline & $\mathrm{R}$ & & S: start of enteral feeding & & \\
\hline & C & & E: non-specified & & \\
\hline
\end{tabular}


Table 1 Studies included in the systematic review and meta-analysis (Continued)

Totsu, 2014 [46]

$P \quad$ Infants with $B W<1500 \mathrm{~g}$

Bifidobacterium bifidum

HM, FM

Dextrin

DB

D: $2.5 \times 10^{9} \mathrm{CFU}$, divided in two doses

CLR

$\mathrm{S}$ : within $48 \mathrm{~h}$ after birth

E: body weight $2000 \mathrm{~g}$

Multic.

$P$ prospective, $D B$ double-blinded, $R$ randomized, $C$ controlled, Multic multicentric, $B$ blinded, $M$ masked, Bic bicentric, BW birth weight, GA gestational age, NEC necrotizing enterocolitis, HM human milk, CFU colony forming unit, $O D$ once daily, $B D$ twice daily, $O M M$ own mother's milk, $P F M$ preterm formula, $D M$ donor milk, $F M$ formula, $M D X$ maltodextrin 
moderate, low and very low (as suggested by the GRADE Working Group) [22].

The association between probiotic use and NEC was evaluated by meta-analyses, conducted by AA and DG, using the RevMan software (version 5.3.5; downloaded from the Cochrane website: http://tech.cochrane.org/ revman/download). Risk ratio (RR) was calculated using the Mantel-Haenszel method, and reported with $95 \%$ confidence interval (CI).

The following sub-meta-analyses were also performed, in order to evaluate the effect of probiotics:

- in specific subgroups of patients (very-low-birth-weight [VLBW] infants);

- in surgical NEC;

- according to NEC incidence in different populations: the incidence of NEC stage $\geq 2$ in the control population was used as a reference, because only a minority of studies reported NEC incidence in the general population. Studies were arbitrarily divided into three groups defined as "low-risk" (NEC incidence $<5 \%$ ), "medium-risk" (incidence 5-10\%), and "high-risk" (incidence $>10 \%$ );

- according to probiotic strain: studies were divided according to the specific probiotic strain used, and were considered as suitable for inclusion in the sub-meta-analyses when the same probiotic strain was used in at least two studies. Studies which used a probiotic mixture were considered together.

Microbiological quality of all the studies was evaluated by MLC and LM. Studies were defined as having severe, moderate or minor microbiological flaws according to the evaluation of proper strain identification and microbiological assessment. Specifically, the lack of proper strain identification was considered as a severe flaw; the lack of microbiological assessment regarding the probiotic persistence in stools was considered as a moderate flaw, whereas a low flaw was defined when the presence of the probiotic in stools was evaluated by indirect approaches such as the quantification of its species belonging.

A fixed-effect model was used for the analyses. Heterogeneity was measured using the $\mathrm{I}^{2}$ test. If significant heterogeneity was present $\left(p<0.05\right.$ from the $\chi^{2}$ test), a random-effects model was used [23]. The randomeffects model was also used when heterogeneity was not significant but the number of studies was $\leq 5$, because the test for heterogeneity is known to have low power when the number of studies is small [24].

Forest plots were used to illustrate results from metaanalyses, and funnel plots to investigate bias.

The online version of GraphPad Quickcalcs software was used to calculate number needed to treat (NNT).

\section{Results}

\section{Literature search}

Three-hundred-sixty-eight papers were identified through the literature search (310 through PubMed and 58 through the Cochrane Library). Thirty-eight studies met the inclusion criteria: 22 were identified through the PubMed search [25-48] and 16 through the Cochrane Library search $[25,26,28-32,34-37,40-43,45]$. Ten additional papers were identified from the reference lists of included studies [49-58]. Of these 47 studies, 16 were excluded, as they were duplicates retrieved both by PubMed and Cochrane Library search. Six additional studies were excluded after examining the full-texts: one study reported maternal probiotic supplementation during pregnancy [29], one cohort was reported twice [31], one study included both term and preterm infants [36], two studies did not report NEC data [55, 56], and in one study randomization was not declared [54].

Twenty-six studies were suitable for inclusion in the meta-analysis $[10,25-28,30,32-35,37-42,44,46,48-$ 53, 58]. A description of included studies is provided in Table 1; excluded studies are described in Table 2.

All the studies reported NEC data in a form suitable for meta-analysis, except one [53], for which data included in a previous Cochrane review were used [59].

Table 2 Studies excluded from the systematic review and meta-analysis

\begin{tabular}{lll}
\hline Author, year & Study summary & Reason for exclusion \\
\hline Awad, 2010 & $\begin{array}{l}\text { Living vs. killed Lactobacillus acidophilus vs. placebo given to neonates admitted } \\
\text { to the study NICU }\end{array}$ & Term and preterm infants included \\
Benor, 2014 & $\begin{array}{l}\text { Lactobacillus acidophilus and Bifidobacteria lactis vs. placebo given to mothers } \\
\text { of VLBW infants }\end{array}$ & Maternal probiotic supplementation \\
Li, 2004 & Bifidobacterium breve given to LBW infants & Randomization not declared \\
Millar, 1993 & Lactobacillus GG given to preterm infants with GA $<33 \mathrm{w}$ & No NEC data \\
Reuman, 1986 & Formula containing lactobacilli vs. placebo given to preterm infants & No NEC data \\
Sari, 2012 & Lactobacillus sporogenes given to preterm infants with GA $<32$ w or BW $<1500 \mathrm{~g}$, & Duplicate population (Sari, 2011 [34]) \\
\hline
\end{tabular}

NICU neonatal intensive care unit, VLBW very low birth weight, $L B W$ low birth weight, GA gestational age, NEC necrotizing enterocolitis, $B W$ birth weight 
Table 3 Incidence of necrotizing enterocolitis in infants treated with probiotics and in controls

\begin{tabular}{|c|c|c|c|c|}
\hline Author, year & Previous NEC rate & Number of subjects & NEC in probiotic group & NEC in control group \\
\hline \multirow[t]{4}{*}{ Al-Hosni, 2012 [33] } & \multirow[t]{4}{*}{ Not stated } & 50 probiotic & $3 / 50$ any stage & $4 / 51$ any stage \\
\hline & & \multirow[t]{3}{*}{51 control } & $1 / 50$ stage 1 & $2 / 51$ stage 1 \\
\hline & & & $0 / 50$ stage 2 & $0 / 51$ stage 2 \\
\hline & & & $2 / 50$ stage 3 & 2/51 stage 3 \\
\hline \multirow[t]{4}{*}{ Bin-Nun, 2005 [40] } & \multirow[t]{4}{*}{$15 \%$} & 72 probiotic & $3 / 72$ any stage & $12 / 73$ any stage \\
\hline & & 73 control & $1 / 72$ stage $\geq 2$ & $10 / 73$ stage $\geq 2$ \\
\hline & & & $1 / 72$ stage 2 & $7 / 73$ stage 2 \\
\hline & & & $0 / 72$ stage 3 & $3 / 73$ stage 3 \\
\hline \multirow[t]{2}{*}{ Braga, 2011 [35] } & \multirow[t]{2}{*}{$10 \%$} & 119 probiotic & \multirow[t]{2}{*}{$0 / 119$ stage $\geq 2$} & \multirow[t]{2}{*}{$4 / 112$ stage $\geq 2$} \\
\hline & & 112 placebo & & \\
\hline \multirow[t]{2}{*}{ Costalos, 2003 [49] } & \multirow[t]{2}{*}{ Not stated } & 51 probiotic & \multirow[t]{2}{*}{$5 / 51$ any stage } & \multirow[t]{2}{*}{$6 / 36$ any stage } \\
\hline & & 36 placebo & & \\
\hline \multirow[t]{2}{*}{ Dani, 2002 [42] } & \multirow[t]{2}{*}{ Not stated } & 295 probiotic & \multirow[t]{2}{*}{$4 / 295$ stage $\geq 2$} & \multirow[t]{2}{*}{$8 / 290$ stage $\geq 2$} \\
\hline & & 290 placebo & & \\
\hline \multirow[t]{2}{*}{ Demirel, 2013 [28] } & \multirow[t]{2}{*}{$32 \%$} & 135 probiotic & \multirow[t]{2}{*}{$6 / 135$ stage $\geq 2$} & \multirow[t]{2}{*}{$7 / 136$ stage $\geq 2$} \\
\hline & & 136 control & & \\
\hline \multirow[t]{2}{*}{ Dilli, 2015 [44] } & \multirow[t]{2}{*}{ Not stated } & 100 probiotic & \multirow[t]{2}{*}{$2 / 100$ stage $\geq 2$} & \multirow[t]{2}{*}{$18 / 100$ stage $\geq 2$} \\
\hline & & 100 placebo & & \\
\hline Fernández-Carrocera, 2013 [32] & $20 \%$ & 75 probiotic & $6 / 75$ stage $\geq 2$ & $12 / 75$ stage $\geq 2$ \\
\hline & & 75 placebo & & \\
\hline Jacobs, 2013 [26] & Not stated & 548 probiotic & $11 / 548$ stage $\geq 2$ & $24 / 551$ stage $\geq 2$ \\
\hline & & 551 placebo & & \\
\hline Kitajima, 1997 [52] & Not stated & 45 probiotic & 0/45 any stage & 0/46 any stage \\
\hline & & 46 placebo & & \\
\hline Lin, 2005 [41] & Approx. $23 \%$ & 180 probiotic & $2 / 180$ stage $\geq 2$ & $10 / 187$ stage $\geq 2$ \\
\hline & (NEL or death) & 187 control & 2/180 stage 2 & 4/187 stage 2 \\
\hline & & & 0/180 stage 3 & 6/187 stage 3 \\
\hline Lin, 2008 [37] & Approx. & 217 placebo & $4 / 217$ any stage & $14 / 217$ any stage \\
\hline & & 217 control & 2/217 stage 2 & 9/217 stage 2 \\
\hline & & & 2/217 stage 3 & $5 / 217$ stage 3 \\
\hline Manzoni, 2006 [37] & Not stated & 39 probiotic & $1 / 39$ any stage & $3 / 41$ any stage \\
\hline & & 41 control & $1 / 39$ stage 2 & $2 / 41$ stage 2 \\
\hline & & & $0 / 39$ stage 3 & 1/41 stage 3 \\
\hline Mihatsch, 2010 [43] & Not stated & 84 probiotic & $2 / 84$ stage $\geq 2$ & $4 / 82$ stage $\geq 2$ \\
\hline & & 82 placebo & & \\
\hline Mohan, 2006 [53] & Not stated & 21 probiotic & $2 / 37$ stage $\geq 2$ & $1 / 32$ stage $\geq 2$ \\
\hline & & 17 placebo & $\begin{array}{l}\text { Unpublished data, taken } \\
\text { from Alfaleh } 2011 \text { [58] }\end{array}$ & $\begin{array}{l}\text { Unpublished data, taken } \\
\text { from Alfaleh } 2011 \text { [58] }\end{array}$ \\
\hline Oncel, 2013 [25] & $15 \%$ & 200 probiotic & $8 / 200$ stage $\geq 2$ & $10 / 200$ stage $\geq 2$ \\
\hline & & 200 placebo & & \\
\hline Patole, 2014 [45] & Not stated & 74 probiotic & $0 / 74$ stage $\geq 2$ & $1 / 66$ stage $\geq 2$ \\
\hline & & 66 placebo & & \\
\hline Rojas, 2012 [30] & Not stated & 372 probiotic & NEC stage $\geq 2$ & NEC stage $\geq 2$ \\
\hline & & 378 placebo & $\leq 1500 \mathrm{~g}$ & $\leq 1500 \mathrm{~g}$ \\
\hline
\end{tabular}


Table 3 Incidence of necrotizing enterocolitis in infants treated with probiotics and in controls (Continued)

\begin{tabular}{|c|c|c|c|c|}
\hline & & & $6 / 176$ probiotic & 10/184 placebo \\
\hline & & & $>1500 \mathrm{~g}$ & $>1500 \mathrm{~g}$ \\
\hline & & & 3/196 probiotic & 5/194 placebo \\
\hline \multirow[t]{2}{*}{ Rougé, 2009 [50] } & Not stated & 45 probiotic & $2 / 45$ any stage & $1 / 49$ any stage \\
\hline & & 49 placebo & & \\
\hline \multirow[t]{2}{*}{ Roy, 2014 [58] } & Not stated & 56 probiotic & $2 / 56$ any stage & $2 / 56$ any stage \\
\hline & & 56 placebo & & \\
\hline \multirow[t]{2}{*}{ Saengtawesin, 2014 [48] } & Not stated & 31 probiotic & $1 / 31$ stage $\geq 2$ & $1 / 29$ stage $\geq 2$ \\
\hline & & 29 placebo & & \\
\hline \multirow[t]{2}{*}{ Samanta, 2009} & Not stated & 91 probiotic & $5 / 91$ stage $\geq 2$ & $15 / 95$ stage $\geq 2$ \\
\hline & & 95 control & & \\
\hline \multirow[t]{3}{*}{ Sari, 2011 [34] } & Approx. $32 \%$ & 110 probiotic & $6 / 110$ stage $\geq 2$ & $10 / 111$ stage $\geq 2$ \\
\hline & & 111 control & $4 / 110$ stage 2 & $7 / 111$ stage 2 \\
\hline & & & 2/110 stage 3 & $3 / 111$ stage 3 \\
\hline \multirow[t]{2}{*}{ Serce, 2013 [27] } & $17 \%$ & 104 probiotic & $7 / 104$ stage $\geq 2$ & $7 / 104$ stage $\geq 2$ \\
\hline & & 104 placebo & & \\
\hline \multirow[t]{2}{*}{ Stratiki, 2007 [39] } & Not stated & 41 probiotic & $0 / 41$ stage $\geq 2$ & $3 / 34$ stage $\geq 2$ \\
\hline & & 34 control & & \\
\hline \multirow[t]{2}{*}{ Totsu, 2014 [46] } & Not stated & 153 probiotic & $0 / 153$ stage $\geq 1$ & $0 / 130$ stage $\geq 1$ \\
\hline & & 130 control & & \\
\hline
\end{tabular}

NEC necrotizing enterocolitis

For each study, NEC rate in the probiotic and in the placebo/control group is reported in Table 3 . For the purpose of the meta-analysis, data on NEC stage $\geq 2$ were used.

\section{Probiotics and NEC stage $\geq \mathbf{2}$}

Data from 6605 infants (3324 in the probiotic group and 3281 in the control group) were analyzed. Fewer infants in the probiotic group developed NEC stage $\geq 2$ compared to infants in the control group (88 [2.65 \%] vs. 188 [5.73 \%], respectively). The RR was significantly lower in infants treated with probiotics (0.47 [95 \% CI 0.36-0.60], $p<0.00001$; fixed-effect analysis). NNT was 33 (95\% CI 24.7-47.2), which means that 33 infants needed to be treated with probiotics in order to prevent one more case of NEC stage $\geq 2$. Heterogeneity among trials was absent $\left(\mathrm{I}^{2}=0 \%, p=0.63\right.$; Fig. $\left.2 \mathrm{a}\right)$. The funnel plot did not show any clear asymmetry (Fig. 2b).

\section{VLBW infants}

Twenty-two studies [25-28, 30, 32-35, 37, 38, 40-42, $44-46,48,50-52]$ reported data from 5912 VLBW infants, 2959 in the probiotic and 2953 in the control group. NEC stage $\geq 2$ occurred less frequently in the probiotic group than in controls (82 [2.77\%] infants vs. 174 [5.89\%], respectively), with a RR of 0.48 ([95 \% CI 0.37$0.62], p<0.00001$; fixed-effect analysis; $\mathrm{I}^{2}=0 \%, p=$ 0.56). NNT was 33 (95\% CI 24.1-47.9).

\section{Surgical NEC}

Only 6 studies [33, 34, 37, 40, 41, 51] reported separate data for surgical NEC (NEC stage 3 ), which occurred in $6 / 668(0.90 \%)$ infants in the probiotic group and in $20 /$ 680 (2.94 \%) infants in the control group. The RR for NEC stage 3 was significantly lower in the probiotic group $(0.35$ [95\% CI 0.16-0.81], $p=0.01$; fixed-effect analysis; $\mathrm{I}^{2}=$ $0 \%, p=0.69)$. NNT was 49 (95\% CI 28.6-170.8).

\section{NEC incidence}

NEC incidence in controls was $<5 \%$ in 13 studies (Fig. 3a) [26, 30, 33, 35, 42, 43, 45, 46, 48, 50, 52, 53, 58], between 5 and $10 \%$ in 8 studies (Fig. 3b) [25, 27, 28, 34, 37, 39, 41, 51], and $>10 \%$ in 5 studies (Fig. 3c) [32, 38, 40, 44, 49].

The RR for NEC stage $\geq 2$ was significantly lower in the probiotic group compared to the control group in all the three populations (RR 0.52 [95 \% CI 0.35-0.78], $p=$ 0.001; RR 0.54 [95 \% CI 0.36-0.80], $p=0.002$; RR 0.33 [95 \% CI $0.17-0.62$ ], $p=0.0006$, respectively). Heterogeneity was non-significant in all the three sub-analyses.

\section{Probiotic strain}

Lactobacillus GG was used in 2 studies [42, 51] and Lactobacillus reuteri in 2 other studies [25, 30]: the effect of these probiotics in reducing NEC was not significant, either for Lactobacillus GG and Lactobacillus 


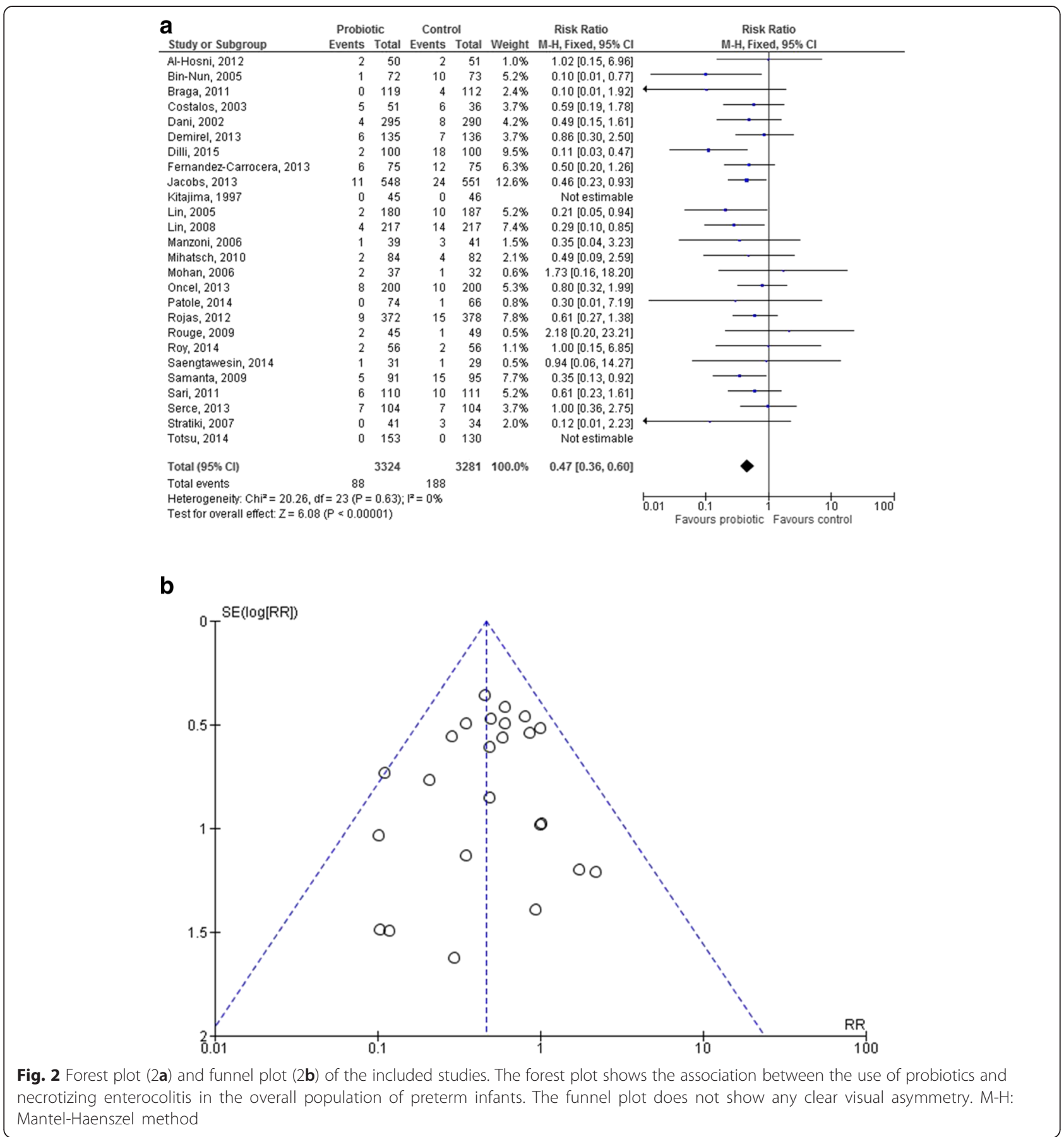

reuteri (RR 0.50 [95 \% CI 0.17-1.44], $p=0.20$ [Fig. 4a], and RR 0.69 [95 \% CI 0.38-1.26], $p=0.23$ [Fig. 4b]). One study used Lactobacillus sporogenes [34].

The results of all the studies including Lactobacilli were pooled, except for the study by Sari et al. [34]: Lactobacillus sporogenes is a species which has not an international recognition, shows characteristics of both genera Lactobacillus and Bacillus, and its strains should be better classified as Bacillus coagulans [60]. Thus, when the results of studies using Lactobacillus GG and reuteri were pooled, no significant reduction in the RR for NEC in the probiotic group was observed (0.62 [95 $\%$ CI 0.37-1.05], $p=0.07$, Fig. 4c).

Four studies used Bifidobacterium lactis [39, 43, 44, 53], 2 studies Bifidobacterium breve [45, 52] and 1 study Bifidobacterium bifidum [46]. The use of 


\begin{tabular}{|c|c|c|c|c|c|c|}
\hline \multirow[b]{2}{*}{ Study or Subgroup } & \multicolumn{2}{|c|}{ Probiotic } & \multicolumn{2}{|c|}{ Control } & \multirow[b]{2}{*}{ Weight } & \multirow{2}{*}{$\begin{array}{c}\text { Risk Ratio } \\
\text { M-H, Fixed, } 95 \%\end{array}$} \\
\hline & Events & Total & Events & Total & & \\
\hline Al-Hosni, 2012 & 2 & 50 & 2 & 51 & $2.8 \%$ & $1.02[0.15,6.9$ \\
\hline Braga, 2011 & 1 & 72 & 10 & 73 & $14.3 \%$ & $0.10[0.01,0$ \\
\hline Dani, 2002 & 4 & 295 & 8 & 290 & $11.6 \%$ & $0.49[0.15,1$ \\
\hline Jacobs, 2013 & 11 & 548 & 24 & 551 & $34.4 \%$ & $0.46[0.23,0$ \\
\hline Kitajima, 1997 & 0 & 45 & 0 & 46 & & Not estim \\
\hline Minatsch, 2010 & 2 & 84 & 4 & 82 & $5.8 \%$ & $0.49[0.09$ \\
\hline Mohan, 2006 & 2 & 37 & 1 & 32 & $1.5 \%$ & $1.73[0.16,18$ \\
\hline Patole, 2014 & 0 & 74 & 1 & 66 & $2.3 \%$ & $0.30[0.01,7$ \\
\hline Rojas, 2012 & 9 & 372 & 15 & 378 & $21.4 \%$ & $0.61[0.27,1$ \\
\hline Rouge, 2009 & 2 & 45 & 1 & 49 & $1.4 \%$ & $2.18[0.20,2]$ \\
\hline Roy, 2014 & 2 & 56 & 2 & 56 & $2.9 \%$ & $1.00[0.15$ \\
\hline Saengtawesin, 2014 & 1 & 31 & 1 & 29 & $1.5 \%$ & $0.94[0.06,1$ \\
\hline Totsu, 2014 & 0 & 153 & 0 & 130 & & Not estim \\
\hline Total $(95 \% \mathrm{Cl})$ & & 1862 & & 1833 & $100.0 \%$ & $0.52[0.35,0$ \\
\hline Total events & 36 & & 69 & & & \\
\hline
\end{tabular}

Heterogeneity: $C \mathrm{Ch}^{2}=6.36, \mathrm{df}=10(\mathrm{P}=0.78) ; \mathrm{I}^{2}=0 \%$

Test for overall effect: $Z=3.20\langle P=0.001\}$

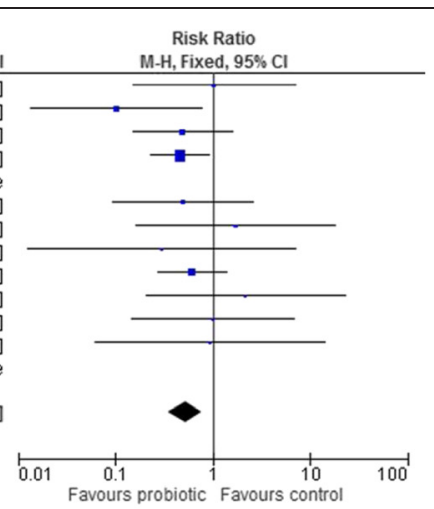

b

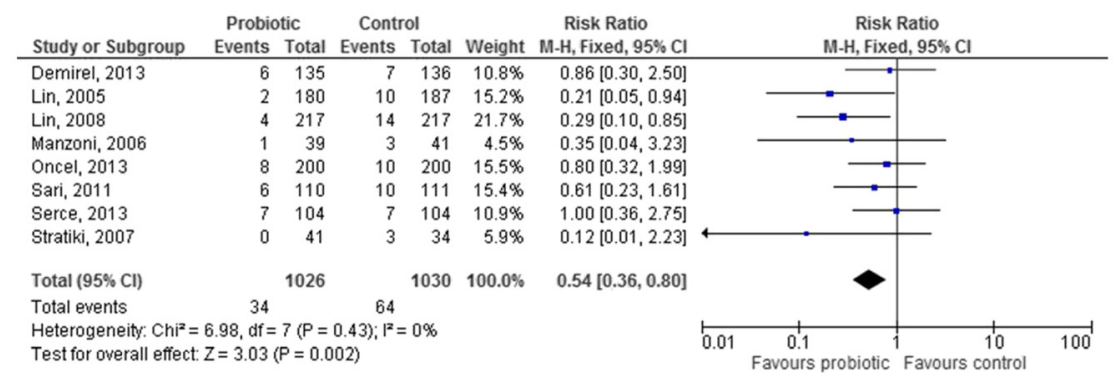

C

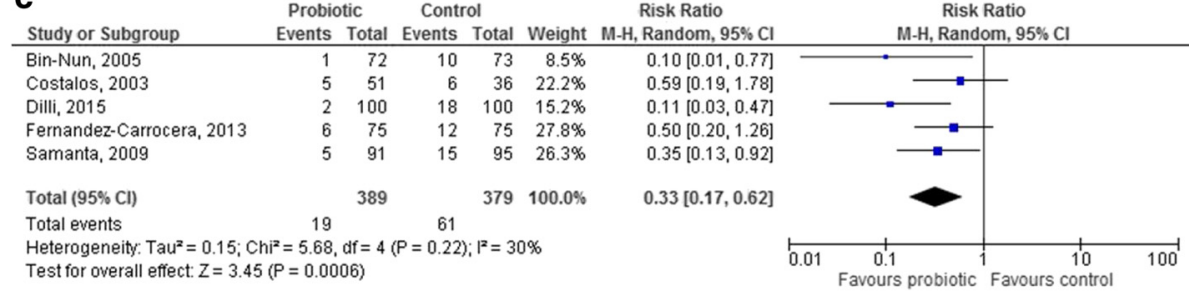

Fig. 3 Forest plot showing the association between the use of probiotics and necrotizing enterocolitis (NEC), according to NEC incidence: (3a). NEC incidence < 5 \%; (3b). NEC incidence 5-10\%; (3c). NEC incidence >10\%. M-H: Mantel-Haenszel method

Bifidobacterium lactis resulted in a significant reduction in the RR for NEC (0.23 [95\% CI 0.10-0.55], $p=$ 0.0008 , Fig. 5a). No effect of Bifidobacterium breve in reducing NEC was documented (RR 0.30 [95 \% CI 0.017.19], $p=0.46$, Fig. 5b); the only study reporting the use of Bifidobacterium bifidum did not report any case on NEC. When the results of studies using Bifidobacteria were pooled, a significant reduction in the RR for NEC in the probiotic group was observed $(0.24$ [95 \% CI 0.10-0.54], $p=0.0006$, Fig. 5c).

Saccharomyces boulardii was used in 3 studies [27, 28, 49]: no significant effect of this probiotic was documented (RR 0.81 [95 \% CI 0.44-1.49], $p=0.50$; random effects analysis).

The pooled analysis of the 11 studies [26, 32, 33, 35, $37,38,40,41,48,50,58]$ in which a probiotic mixture was used showed an overall and significant benefit of these products in reducing NEC (RR 0.39 [95 \% CI 0.27-0.56], $p<0.00001$, Fig. 6).

\section{Study quality}

Evaluation of the quality of the studies included in the meta-analysis according to the risk of bias tool as proposed by the Cochrane Collaboration is showed in Table 4, which also shows the level of evidence evaluated following the recommendations of the GRADE Working Group.

\section{Microbiological quality}

Microbiological quality of included studies is described in Table 5. Eight studies were evaluated as having severe microbiological flaws [27, 32, 34, 35, 38, 40, 41, 49], meaning that they did not report a proper probiotic strain identification. Thirteen studies $[25,26,28,30,33$, $37,42-44,46,48,51,58]$ were evaluated as having moderate microbiological flaws, because none of them evaluated the probiotic persistence in stools. There were only five studies [39, 45, 50, 52, 53] with minor microbiological flaws. 

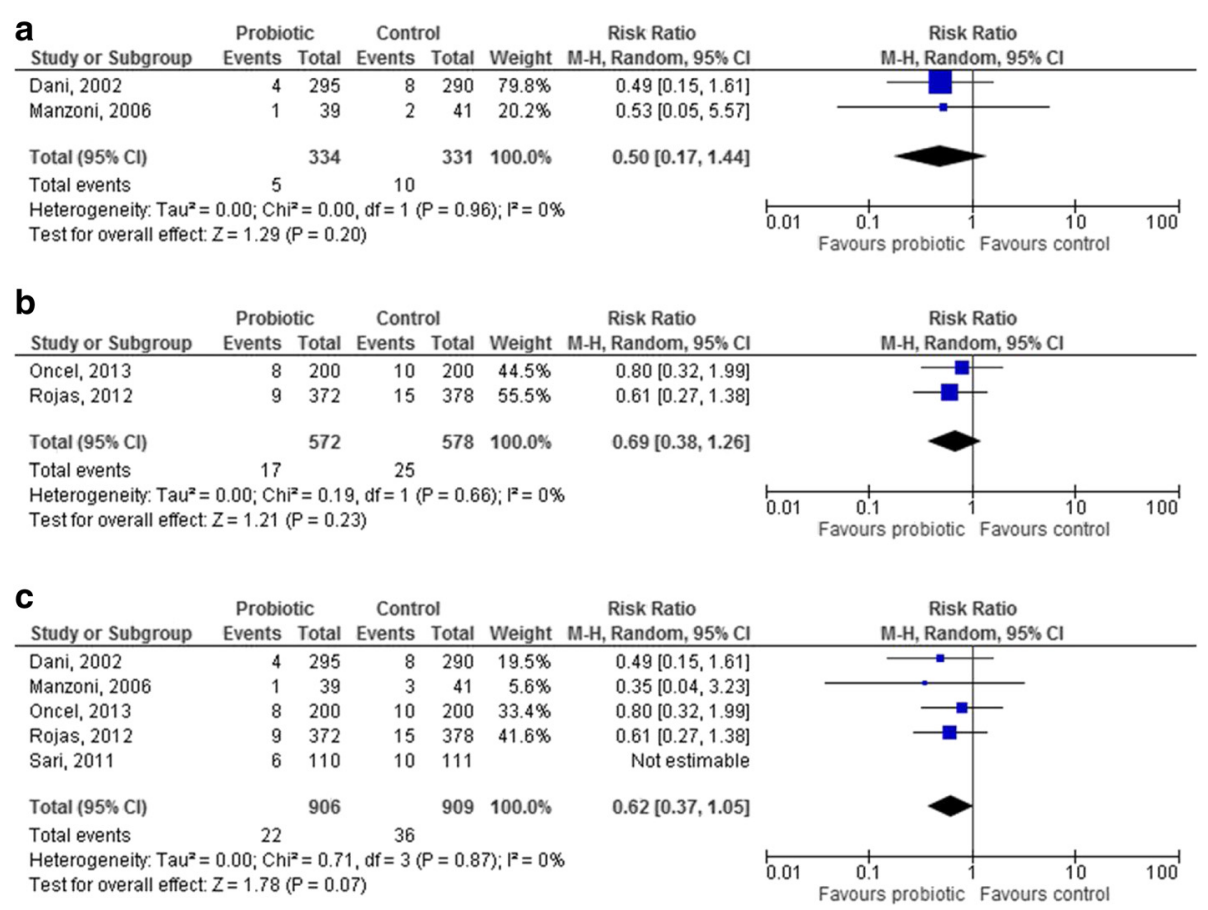

Fig. 4 Forest plot showing the association between probiotics and necrotizing enterocolitis in the studies which used a single-strain product containing Lactobacilli ((4a). L. reuteri; (4b). L. GG; (4c). pooled analysis of all the studies using Lactobacilli). M-H: Mantel-Haenszel method

\section{Discussion}

The results of this systematic review and meta-analysis show an overall benefit of probiotic supplementation for the prevention of NEC in preterm infants. These results are strengthened by the absence of significant statistical heterogeneity among studies and by the low-risk of publication bias documented by the funnel plot.

However, despite the overall benefit, it is remarkable that the 26 studies included in the meta-analysis were extremely heterogeneous in terms of probiotic strain, dosage, duration of intervention, and target population. Furthermore, only few studies documented an effective colonization of the infants' gut with the probiotic strain. Thus, the proposal made by the authors of the recent Cochrane review of a "change in practice" in the use of probiotics in preterm infants [11] might require further investigation.

Currently available literature does not provide any definite conclusion on which probiotic strain should be used, and which group of preterm infants would benefit most from a probiotic intervention. It is important to note that the effect of a live-microorganism used as a probiotic is strictly strain-specific [61]. In this paper we aimed to perform strain-specific sub-meta-analyses but our efforts were weakened by the fact that in very few studies the same probiotic strain was used. For this reason, we were unable to draw definite conclusions on which single-strain of probiotics would be more effective in reducing NEC. When studies using single strains were pooled according to the probiotic genus, no significant effect was documented for Lactobacilli and Saccharomyces. This is partially in contrast with the recent Cochrane review on probiotics and NEC [11], which showed a beneficial effect of Lactobacilli: this discrepancy appears to be due mainly to differences in the studies included in the two sub-meta-analyses. Actually, the present meta-analysis included the study by Oncel et al. [25], which was on-going when the Cochrane review was published, but excluded the study by Manzoni et al. [57], where probiotics were used in addition to lactoferrin, and the study by Sari et al. [34], which used a probiotic product which is not properly a Lactobacillus [60].

The analysis of studies using Bifidobacteria showed a significant effect of Bifidobacterium breve in reducing NEC. This is also in contrast with the results of the Cochrane review; however, the discrepancy is explained by the inclusion in the present meta-analysis of the recent study by Dilli et al. [44], which appears to drive the beneficial effect documented for Bifidobacteria. Similarly to the Cochrane review [11], the analysis of studies in which more than one strain was used documented a strong and significant effect of these products in the prevention of NEC. No definite conclusion can be drawn from these results, even if it could be suggested that further research should be focused on mixed rather than on single-strain products; a potential rationale for this 


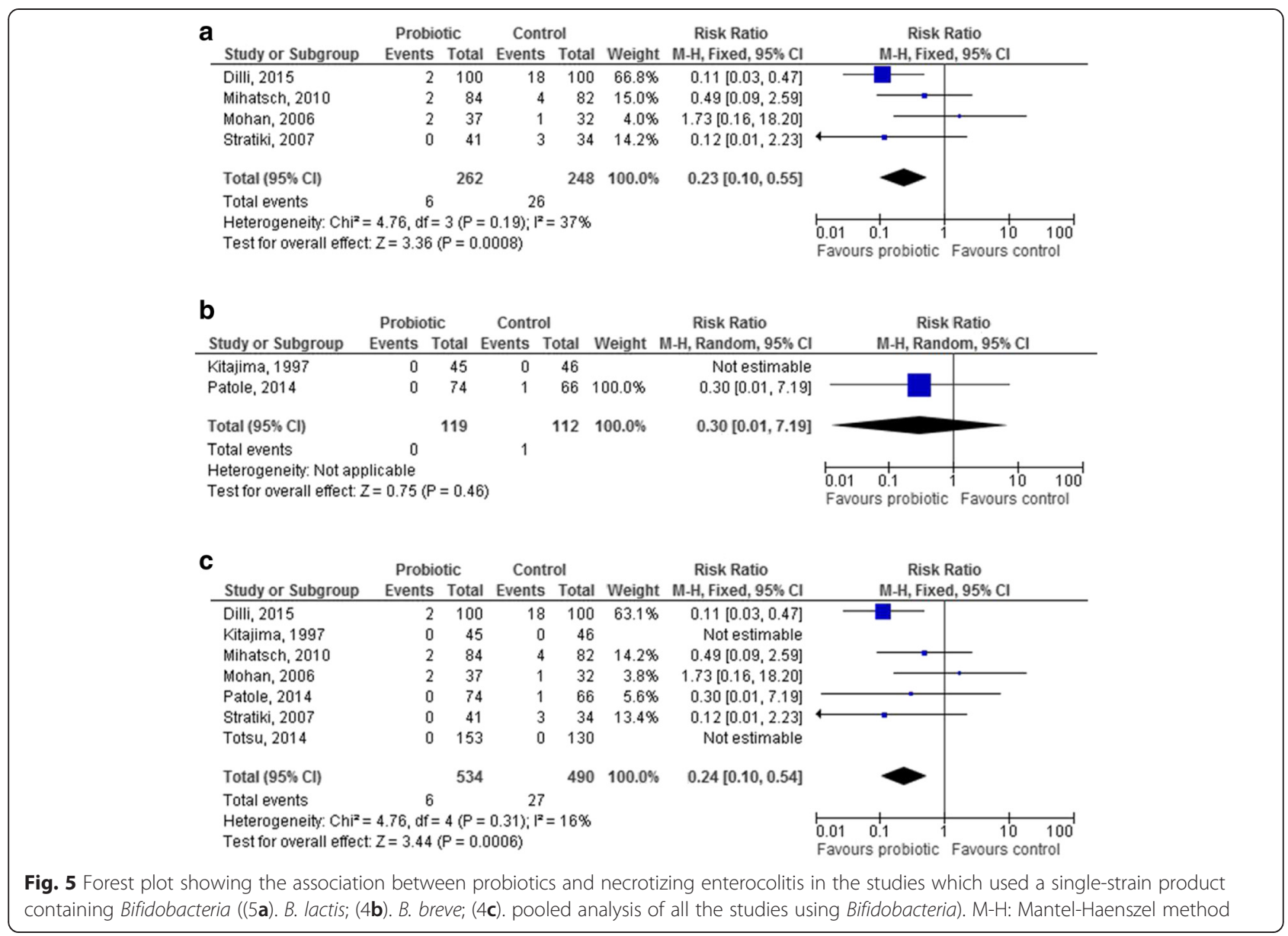

approach could be that a mix of strains might be more effective in providing an ecological barrier than a single strain.

The evidence that probiotics are effective in reducing NEC in VLBW infants does not necessarily apply also to extremely LBW infants (ELBWs), who are the highestrisk population. Only three studies [26, 33, 58] reported the rate of NEC in ELBWs: in two of these studies
$[33,58]$, the same number of ELBWs in the probiotic and control group developed NEC [33], while in the ProPrems trial NEC incidence was slightly lower in the probiotic group [26]. Given the relatively small number of ELBWs and the inconclusive results, no specific recommendation can be drawn from the analysis of these two studies. Similarly, no study reported separate data for intrauterine-growth-restricted (IUGR) infants, and thus

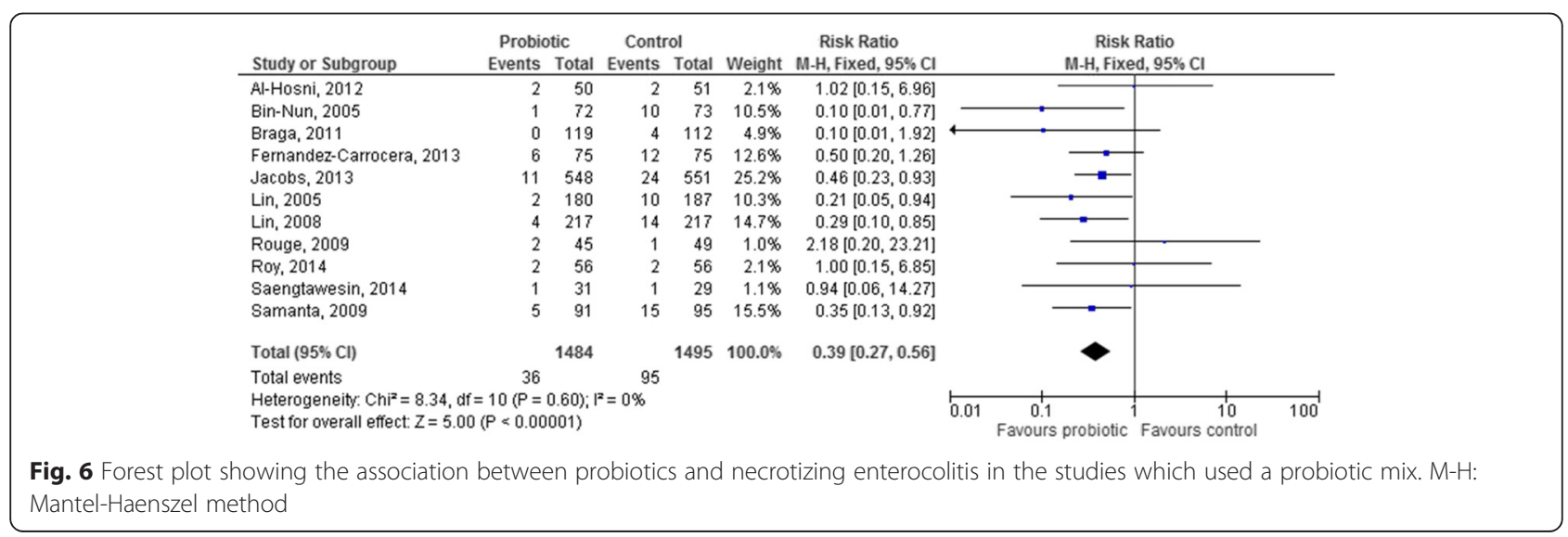


Table 4 Evaluation of the quality of the studies included in the meta-analysis according to the risk of bias tool as proposed by the Cochrane collaboration and evaluation of the level of evidence according to the GRADE approach

\begin{tabular}{|c|c|c|c|c|c|c|c|}
\hline Study & $\begin{array}{l}\text { Random sequence } \\
\text { generation }\end{array}$ & $\begin{array}{l}\text { Allocation } \\
\text { concealment }\end{array}$ & Blinding & $\begin{array}{l}\text { Incomplete } \\
\text { outcome data }\end{array}$ & $\begin{array}{l}\text { Selective outome } \\
\text { reporting }\end{array}$ & $\begin{array}{l}\text { Other sources } \\
\text { of bias }\end{array}$ & $\begin{array}{l}\text { Levels of quality of evidence } \\
\text { in the grade approach }\end{array}$ \\
\hline Al-Hosni, 2012 [33] & UNCLEAR & UNCLEAR & LOW & UNCLEAR & UNCLEAR & UNCLEAR & LOW \\
\hline Bin-Nun, 2005 [40] & UNCLEAR & UNCLEAR & $\mathrm{HIGH}$ & UNCLEAR & UNCLEAR & UNCLEAR & VERYLOW \\
\hline Braga, 2011 [35] & LOW & LOW & LOW & LOW & UNCLEAR & LOW & $\mathrm{HIGH}$ \\
\hline Costalos, 2003 [49] & LOW & LOW & LOW & LOW & UNCLEAR & LOW & $\mathrm{HIGH}$ \\
\hline Dani, 2002 [42] & UNCLEAR & LOW & LOW & LOW & UNCLEAR & UNCLEAR & MODERATE \\
\hline Demirel, 2013 [28] & LOW & LOW & LOW & UNCLEAR & UNCLEAR & UNCLEAR & MODERATE \\
\hline Dilli, 2015 [44] & LOW & LOW & LOW & UNCLEAR & UNCLEAR & UNCLEAR & MODERATE \\
\hline $\begin{array}{l}\text { Fernández-Carrocera, } \\
2013 \text { [32] }\end{array}$ & LOW & LOW & LOW & LOW & UNCLEAR & LOW & $\mathrm{HIGH}$ \\
\hline Jacobs, 2013 [26] & LOW & UNCLEAR & LOW & UNCLEAR & UNCLEAR & UNCLEAR & LOW \\
\hline Kitajima, 1997 [52] & LOW & UNCLEAR & LOW & UNCLEAR & UNCLEAR & LOW & MODERATE \\
\hline Lin, 2005 [41] & LOW & LOW & LOW & LOW & UNCLEAR & LOW & $\mathrm{HIGH}$ \\
\hline Lin, 2008 [37] & LOW & LOW & LOW & LOW & UNCLEAR & LOW & $\mathrm{HIGH}$ \\
\hline Manzoni, 2006 [37] & LOW & LOW & LOW & UNCLEAR & UNCLEAR & LOW & MODERATE \\
\hline Mihatsch, 2010 [43] & LOW & UNCLEAR & LOW & LOW & UNCLEAR & LOW & MODERATE \\
\hline Mohan, 2006 [53] & UNCLEAR & LOW & LOW & UNCLEAR & UNCLEAR & UNCLEAR & LOW \\
\hline Oncel, 2013 [25] & LOW & UNCLEAR & LOW & LOW & UNCLEAR & UNCLEAR & MODERATE \\
\hline Patole, 2014 [45] & LOW & LOW & LOW & LOW & UNCLEAR & LOW & $\mathrm{HIGH}$ \\
\hline Rojas, 2012 [30] & LOW & LOW & LOW & LOW & UNCLEAR & LOW & $\mathrm{HIGH}$ \\
\hline Rougé, 2009 [50] & LOW & UNCLEAR & UNCLEAR & UNCLEAR & UNCLEAR & $\mathrm{HIGH}$ & LOW \\
\hline Roy, 2014 [58] & LOW & UNCLEAR & LOW & LOW & UNCLEAR & UNCLEAR & MODERATE \\
\hline $\begin{array}{l}\text { Saengtawesin, } 2014 \\
\text { [48] }\end{array}$ & $\mathrm{HIGH}$ & $\mathrm{HIGH}$ & $\mathrm{HIGH}$ & UNCLEAR & UNCLEAR & UNCLEAR & LOW \\
\hline Samanta, 2009 & LOW & LOW & LOW & UNCLEAR & UNCLEAR & UNCLEAR & MODERATE \\
\hline Sari, 2011 [34] & LOW & LOW & LOW & UNCLEAR & UNCLEAR & UNCLEAR & MODERATE \\
\hline Serce, 2013 [27] & LOW & LOW & LOW & UNCLEAR & UNCLEAR & LOW & MODERATE \\
\hline Stratiki, 2007 [39] & UNCLEAR & UNCLEAR & LOW & UNCLEAR & UNCLEAR & LOW & LOW \\
\hline Totsu, 2014 [46] & LOW & LOW & LOW & LOW & UNCLEAR & UNCLEAR & MODERATE \\
\hline
\end{tabular}

no recommendation can be made either for this highrisk population.

In the analysis of trials evaluating a specific intervention, it is pivotal to understand whether the results of these trials are generalizable or applicable only in specific clinical settings. According to our data, the common belief that probiotics are more effective in populations with a high rate of NEC [62] can be called into question: actually, when studies were divided according to NEC incidence in the control population, NEC reduction was striking and significant also when NEC rate in controls was extremely low. NEC rate in controls can be considered as a proxy for the quality of neonatal care: in this perspective, it is interesting to note that, in contrast with previous data, probiotics appear to confer a preventive benefit also in high quality-of-care settings. NEC rate in control populations was used for the analysis, rather than the baseline NEC rate stated by the authors and used in several studies for sample size calculation: this approach was considered more appropriate, because baseline NEC rate was not provided in many studies and, when provided, there was often a discrepancy with NEC rate detected in controls.

The analysis of included studies according to their microbiological quality points out that clinical studies aiming at evaluate the preventive effect of probiotics on NEC often lack an adequate microbiological assessment and this represents a major limitation of these studies. Actually, it is well known that the correct identification of a probiotic at species level corresponds to evaluate its safety, whereas the identification at strain level is extremely relevant as probiotic beneficial properties are strain-specific. Furthermore, the evaluation of probiotic colonisation, even if temporary, 
Table 5 Evaluation of the included studies according to their microbiological quality

\begin{tabular}{|c|c|c|c|c|}
\hline Author, year & Probiotic strain & Strain identification & Microbiological assessment & Microbiological flaw \\
\hline \multirow[t]{2}{*}{ Al-Hosni, 2012 [33] } & Lactobacillus rhamnosus LGG & LGG identified at the strain level & No assessment & Moderate \\
\hline & Bifidobacterium infantis & $\begin{array}{l}\text { B. infantis identified via the web site of } \\
\text { the producer: Bifantis (Bifidobacterium } \\
\text { infantis 35624) }\end{array}$ & & \\
\hline \multirow[t]{3}{*}{ Bin-Nun, 2005 [40] } & Bifidobacterium infantis & Strains not identified at the strain level & No assessment & Severe \\
\hline & Streptococcus thermophilus & & & \\
\hline & Bifidobacterium bifidus & & & \\
\hline \multirow[t]{2}{*}{ Braga, 2011 [35] } & Lactobacillus casei & Strains non identified clearly & No assessment & Severe \\
\hline & Bifidobacterium breve & & & \\
\hline \multirow[t]{2}{*}{ Costalos, 2003 [49] } & Saccharomyces boulardii & Strain not identified at the strain level & $\begin{array}{l}\text { S. boulardii not characterized } \\
\text { in stools. }\end{array}$ & Severe \\
\hline & & & $\begin{array}{l}\text { Gut flora assessed by plate } \\
\text { count }\end{array}$ & \\
\hline Dani, 2002 [42] & Lactobacillus rhamnosus GG & Strain identified & No assessment & Moderate \\
\hline Demirel, 2013 [28] & Saccharomyces boulardii & Strain identified & No assessment & Moderate \\
\hline Dilli, 2015 [44] & B. lactis & $\begin{array}{l}\text { Strain non identified at the strain level } \\
\text { but probably Bb12 }\end{array}$ & No assessment & Moderate \\
\hline \multirow{6}{*}{$\begin{array}{l}\text { Fernández-Carrocera, } \\
2013 \text { [32] }\end{array}$} & Lactobacillus acidophilus & Strains not identified at the strain level & No assessment & Severe \\
\hline & Lactobacillus rhamnosus & & & \\
\hline & Lactobacillus casei & & & \\
\hline & Lactobacillus plantarum & & & \\
\hline & Bifidobacterium infantis & & & \\
\hline & Streptococcus thermophilus & & & \\
\hline \multirow[t]{2}{*}{ Jacobs, 2013 [26] } & Bifidobacterium infantis & Strains identified at the strain level & No assessment & Moderate \\
\hline & $\begin{array}{l}\text { Streptococcus thermophilus } \\
\text { Bifidobacterium lactis }\end{array}$ & & & \\
\hline Kitajima, 1997 [52] & Bifidobacterium breve & Strain identified & $\begin{array}{l}\text { Assessment by a strain-specific } \\
\text { monoclonal antibody } \\
\text { conjugated with colloidal } \\
\text { gold particle }\end{array}$ & Minor \\
\hline \multirow[t]{2}{*}{ Lin, 2005 [41] } & Lactobacillus acidophilus & Strains not identified at the strain level & No assessment & Severe \\
\hline & Bifidobacterium infantis & & & \\
\hline \multirow[t]{2}{*}{ Lin, 2008 [37] } & Lactobacillus acidophilus & Strain identified & No assessment & Moderate \\
\hline & Bifidobacterium bifidum & & & \\
\hline Manzoni, 2006 [51] & Lactobacillus rhamnosus LGG & Strain identified & No assessment & Moderate \\
\hline Mihatsch, 2010 [43] & Bifidobacterium lactis & Strain identified & No assessment & Moderate \\
\hline Mohan, 2006 [53] & Bifidobacterium lactis & Strain identified & $\begin{array}{l}\text { Species-specific (not strain- } \\
\text { specific) assessment }\end{array}$ & Minor \\
\hline Oncel, 2013 [25] & Lactobacillus reuteri & Strain identified & No assessment & Moderate \\
\hline Patole, 2014 [45] & Bifidobacterium breve & Strain identified & $\begin{array}{l}\text { Microbiological assessment } \\
\text { by PCR }\end{array}$ & Minor \\
\hline Rojas, 2012 [30] & Lactobacillus reuteri & Strain identified & No assessment & Moderate \\
\hline \multirow[t]{2}{*}{ Rougé, 2009 [50] } & Bifidobacterium longum & Strain identified & \multirow{2}{*}{$\begin{array}{l}\text { Microbiological assessment } \\
\text { by PCR }\end{array}$} & \multirow[t]{2}{*}{ Minor } \\
\hline & Lactobacillus rhamnosus GG & & & \\
\hline Roy, 2014 [58] & $\begin{array}{l}\text { Lactobacillus acidophilus, } \\
\text { B. longum, B. bifidum, and } \\
\text { B. lactis }\end{array}$ & $\begin{array}{l}\text { Strains not identified at the strain } \\
\text { level/identification of the commercial } \\
\text { product }\end{array}$ & No assessment & Moderate \\
\hline Saengtawesin, 2014 [48] & Lactobacillus acidophilus and & & No assessment & Moderate \\
\hline
\end{tabular}


Table 5 Evaluation of the included studies according to their microbiological quality (Continued)

\begin{tabular}{|c|c|c|c|c|}
\hline & & $\begin{array}{l}\text { Strains not identified at the strain } \\
\text { level/identification of the commercial } \\
\text { product }\end{array}$ & & \\
\hline \multirow[t]{4}{*}{ Samanta, 2009} & Bifidobacterium infantis & Strains not identified at the strain level & No assessment & Severe \\
\hline & Bifidobacterium bifidum & & & \\
\hline & Bifidobacterium longum & & & \\
\hline & Lactobacillus acidophilus & & & \\
\hline Sari, 2011 [34] & Lactobacillus sporogenes & Strains not identified at the strain level & No assessment & Severe \\
\hline Serce, 2013 [27] & Saccharomyces boulardii & Strains not identified at the strain level & No assessment & Severe \\
\hline Stratiki, 2007 [39] & Bifidobacterium lactis & Strain identified & $\begin{array}{l}\text { Assessment by plate count, } \\
\text { no strain-specific assessment }\end{array}$ & Minor \\
\hline Totsu, 2014 [46] & Bifidobacterium bifidum & Strain identified & No assessment & Moderate \\
\hline
\end{tabular}

is important to correlate the probiotic presence to the beneficial effects.

The development of gut microbiota in preterm infants is known to be influenced by several factors, including gestational age, mode of delivery, diet, and antibiotic exposure [63]. All these factors are likely to be significant confounders in the relationship between probiotics and NEC: actually, it is well documented that infants fed maternal or donor breast milk have a lower risk of NEC compared to formula-fed infants [64], and that caesarean delivery is associated with a disruption in gut microbiota [65]. Quite surprisingly, however, in published studies data are not analyzed taking these confounders into account [66]. Given the definite protective role of human milk feeding and the symbiotic properties of human milk, it would be fundamental to understand whether the use of probiotics should be encouraged also in human-milk fed infants, or if this intervention should be directed towards exclusively formula-fed infants.

The studies included in the meta-analysis did not report any short-term adverse effect of probiotic supplementation (i.e., bloodstream infection with the probiotic strain). Growing evidence suggests the influence of gut microbiota on long-term health and disease, including both type 1 and type 2 diabetes mellitus, atherosclerosis, asthma, colon cancer, and inflammatory bowel disease [67]. However, at present little is known on the longterm outcome possibly related to the alteration of gut flora in preterm infants, which is the result of the supplementation with exogenous strains.

The choice to investigate a single outcome might be viewed as a limitation of the study: however, this choice was deliberate, as the literature search strategy was focused exclusively on NEC. Any speculation on different outcomes such as sepsis or mortality would have been inevitably misleading, because it would have been impossible to be sure to have identified all the studies reporting on those outcomes.

\section{Conclusions}

Meta-analyses give a valuable contribution in guiding researchers to focus future clinical studies on specific unanswered questions. The results of the present metaanalysis confirm that research on probiotics and NEC is on the right track, but also suggest that there are several unanswered questions which should be addressed before radically changing clinical practice. Our data highlight the need for further, well-designed studies aimed at clarifying the specific effect of probiotics in high-risk populations (i.e., ELBWs, IUGRs) and at addressing the choice of the most effective probiotic product, at the proper dose and duration of supplementation. For this reason, we encourage, for future studies, the publication of study protocols detailing study population and characteristics of the intervention, in order to narrow probiotic research to the most promising strains or combination of strains and to the most vulnerable populations, thus allowing a confirmative individual patient data analysis.

\section{Competing interests}

None of the authors has any conflict of interest to declare in connection with this paper.

\section{Authors' contributions}

All the authors, as part of the Task Force on Probiotics of the Italian Society of Neonatology, conceived and designed the study protocol. AA and LC performed the literature search and assessed study details, which were checked by DG. AA and DG evaluated study quality and performed the meta-analyses. MLC and LM evaluated microbiological quality of the included studies. AA and LC wrote the first draft of the paper, which was critically revised by all the other authors. All the authors gave final approval of the version to be submitted and agreed to be accountable for the whole paper.

\section{Authors' information}

Task Force on Probiotics of the Italian Society of Neonatology: Arianna Aceti, Davide Gori, Giovanni Barone, Maria Luisa Callegari, Antonio Di Mauro, Maria Pia Fantini, Flavia Indrio, Luca Maggio, Fabio Meneghin, Lorenzo Morelli, Gianvincenzo Zuccotti, Luigi Corvaglia.

Study Group of Neonatal Gastroenterology and Nutrition of the Italian Society of Neonatology: Flavia Indrio, Luca Maggio, Luigi Corvaglia. Coordinator of the Task Force on Probiotics of the Italian Society of Neonatology: Luigi Corvaglia. 


\section{Funding}

No funding was received from any of the authors for this paper.

\section{Author details}

${ }^{1}$ Neonatal Intensive Care Unit, Department of Medical and Surgical Sciences (DIMEC), University of Bologna, S.Orsola-Malpighi Hospital, Bologna, Italy. ${ }^{2}$ Department of Biomedical and Neuromotor Sciences (DIBINEM), University of Bologna, Bologna, Italy. ${ }^{3}$ Neonatal Unit, Catholic University, Rome, Italy. ${ }^{4}$ Institute of Microbiology, UCSC, Piacenza, Italy. ${ }^{5}$ Department of Pediatrics, Aldo Moro University, Bari, Italy. ${ }^{6}$ Department of Pediatrics, University of Milan, Luigi Sacco Hospital, Milan, Italy.

\section{Received: 27 July 2015 Accepted: 8 November 2015} Published online: 14 November 2015

\section{References}

1. Neu J, Walker WA. Necrotizing enterocolitis. N Engl J Med. 2011;364:255-64.

2. Bisquera JA, Cooper TR, Berseth CL. Impact of necrotizing enterocolitis on length of stay and hospital charges in very low birth weight infants. Pediatrics. 2002;109:423-8.

3. Gephart S, McGrath J, Effken J, Halpern M. Necrotizing enterocolitis risk: state of the science. Adv Neonatal Care. 2012;12:77-89.

4. Sanders ME, Guarner F, Guerrant R, Holt PR, Quigley EMM, Sartor RB, et al. An update on the use and investigation of probiotics in health and disease. Gut. 2013;62:787-96.

5. Martin CR, Walker WA. Probiotics: role in pathophysiology and prevention in necrotizing enterocolitis. Semin Perinatol. 2008;32:127-37.

6. DiGiulio DB, Romero R, Amogan HP, Kusanovic JP, Bik EM, Gotsch F, et al. Microbial prevalence, diversity and abundance in amniotic fluid during preterm labor: a molecular and culture-based investigation. PLoS One. 2008;3:e3056.

7. Mshvildadze M, Neu J, Shuster J, Theriaque D, Li N, Mai V. Intestinal microbial ecology in premature infants assessed with non-culture-based techniques. J Pediatr. 2010;156:20-5.

8. Wang Y, Hoenig JD, Malin KJ, Qamar S, Petrof EO, Sun J, et al. $16 \mathrm{~S}$ rRNA gene-based analysis of fecal microbiota from preterm infants with and without necrotizing enterocolitis. ISME J. 2009;3:944-54.

9. Murguía-Peniche T, Mihatsch WA, Zegarra J, Supapannachart S, Ding Z-Y, Neu J. Intestinal mucosal defense system, Part 2. Probiotics and prebiotics. J Pediatr. 2013;162(3 Suppl):S64-71.

10. Deshpande G, Rao S, Patole S, Bulsara M. Updated meta-analysis of probiotics for preventing necrotizing enterocolitis in preterm neonates. Pediatrics. 2010;125:921-30

11. Alfaleh K, Anabrees J, Bassler D. Probiotics for prevention of necrotizing enterocolitis in preterm infants. Cochrane Database Syst Rev. 2014;4:CD005496. doi:10.1002/14651858.CD005496.pub4.

12. Thomas DW, Greer FR. Probiotics and prebiotics in pediatrics. Pediatrics. 2010;126:1217-31.

13. Mihatsch WA, Braegger CP, Decsi T, Kolacek S, Lanzinger $H$, Mayer $B$, et al. Critical systematic review of the level of evidence for routine use of probiotics for reduction of mortality and prevention of necrotizing enterocolitis and sepsis in preterm infants. Clin Nutr. 2012;31:6-15.

14. Szajewska H. Probiotics and prebiotics in preterm infants: where are we? Where are we going? Early Hum Dev. 2010;86 Suppl 1:81-6.

15. Hill C, Guarner F, Reid G, Gibson GR, Merenstein DJ, Pot B, et al. Expert consensus document: The International Scientific Association for Probiotics and Prebiotics consensus statement on the scope and appropriate use of the term probiotic. Nat Rev Gastroenterol Hepatol. 2014;11:506-14.

16. FAO, WHO. Probiotics in food. Health and nutritional properties and guidelines for evaluation. 2006

17. Moher D, Liberati A, Tetzlaff J, Altman DG. Preferred reporting items for systematic reviews and meta-analyses: the PRISMA statement. PLoS Med. 2009;6:e1000097.

18. Bell MJ, Ternberg JL, Feigin RD, Keating JP, Marshall R, Barton L, et al. Neonatal necrotizing enterocolitis: therapeutic decisions based upon clinical staging. Ann Surg. 1978;187:1-7.

19. Walsh MC, Kliegman RM. Necrotizing enterocolitis: treatment based on staging criteria. Pediatr Clin North Am. 1986;33:179-201.

20. Greenhalgh T, Peacock R. Effectiveness and efficiency of search methods in systematic reviews of complex evidence: audit of primary sources. BMJ. 2005;331:1064-5.
21. Cochrane handbook for systematic reviews of interventions. [http:// handbook.cochrane.org/].

22. Guyatt GH, Oxman AD, Vist GE, Kunz R, Falck-Ytter Y, Alonso-Coello P, et al. GRADE: an emerging consensus on rating quality of evidence and strength of recommendations. BMJ. 2008;336:924-6.

23. Higgins JPT, Green S, editors. Cochrane handbook for systematic reviews of interventions. Version 5.1.0 (March 2011). 2011.

24. Hardy RJ, Thompson SG. Detecting and describing heterogeneity in meta-analysis. Stat Med. 1998;17:841-56.

25. Oncel MY, Sari FN, Arayici S, Guzoglu N, Erdeve O, Uras N, et al. Lactobacillus Reuteri for the prevention of necrotising enterocolitis in very low birthweight infants: a randomised controlled trial. Arch Dis Child Fetal Neonatal Ed. 2014;99:F110-5.

26. Jacobs SE, Tobin JM, Opie GF, Donath S, Tabrizi SN, Pirotta M, et al. Probiotic effects on late-onset sepsis in very preterm infants: a randomized controlled trial. Pediatrics. 2013;132:1055-62.

27. Serce O, Benzer D, Gursoy T, Karatekin G, Ovali F. Efficacy of saccharomyces boulardii on necrotizing enterocolitis or sepsis in very low birth weight infants: a randomised controlled trial. Early Hum Dev. 2013;89:1033-6.

28. Demirel G, Erdeve O, Celik IH, Dilmen U. Saccharomyces boulardii for prevention of necrotizing enterocolitis in preterm infants: a randomized, controlled study. Acta Paediatr. 2013;102:560-5.

29. Benor S, Marom R, Ben Tov A, Domany KA, Zaidenberg-Israeli G, Dollberg S. Probiotic supplementation in mothers of very low birth weight infants. Am J Perinatol. 2014;31:497-504.

30. Rojas MA, Lozano JM, Rojas MX, Rodriguez VA, Rondon MA, Bastidas JA, et al. Prophylactic probiotics to prevent death and nosocomial infection in preterm infants. Pediatrics. 2012;130:e1113-20.

31. Sari FN, Eras Z, Dizdar EA, Erdeve O, Oguz SS, Uras N, et al. Do oral probiotics affect growth and neurodevelopmental outcomes in very low-birth-weight preterm infants? Am J Perinatol. 2012;29:579-86.

32. Fernández-Carrocera LA, Solis-Herrera A, Cabanillas-Ayón M, GallardoSarmiento RB, García-Pérez CS, Montaño-Rodríguez R, et al. Double-blind, randomised clinical assay to evaluate the efficacy of probiotics in preterm newborns weighing less than $1500 \mathrm{~g}$ in the prevention of necrotising enterocolitis. Arch Dis Child Fetal Neonatal Ed. 2013;98:F5-9.

33. Al-Hosni M, Duenas M, Hawk M, Stewart LA, Borghese RA, Cahoon M, et al. Probiotics-supplemented feeding in extremely low-birth-weight infants. J Perinatol. 2012;32:253-9.

34. Sari FN, Dizdar EA, Oguz S, Erdeve O, Uras N, Dilmen U. Oral probiotics: Lactobacillus sporogenes for prevention of necrotizing enterocolitis in very low-birth weight infants: a randomized, controlled trial. Eur J Clin Nutr. 2011;65:434-9.

35. Braga TD, da Silva GAP, de Lira PI, de Carvalho LM. Efficacy of Bifidobacterium breve and Lactobacillus casei oral supplementation on necrotizing enterocolitis in very-low-birth-weight preterm infants : a double-blind, randomized, controlled trial. Am J Clin Nutr. 2011;93:81-6.

36. Awad H, Mokhtar H, Imam SS, Gad Gl, Hafez H, Aboushady N. Comparison between killed and living probiotic usage versus placebo for the prevention of necrotizing enterocolitis and sepsis in neonates. Pak J Biol Sci. 2010;13:253-62.

37. Lin H-C, Hsu C-H, Chen H-L, Chung M-Y, Hsu J-F, Lien R, et al. Oral probiotics prevent necrotizing enterocolitis in very low birth weight preterm infants: a multicenter, randomized, controlled trial. Pediatrics. 2008;122:693-700.

38. Samanta M, Sarkar M, Ghosh P, Ghosh JK, Sinha MK, Chatterjee S. Prophylactic probiotics for prevention of necrotizing enetrocolitis in very low birth weight newborns. J Trop Pediatr. 2008;55:128-31.

39. Stratiki Z, Costalos C, Sevastiadou S, Kastanidou O, Skouroliakou M, Giakoumatou A, et al. The effect of a bifidobacter supplemented bovine milk on intestinal permeability of preterm infants. Early Hum Dev. 2007:83:575-9.

40. Bin-Nun A, Bromiker R, Wilschanski M, Kaplan M, Rudensky B, Caplan M, et al. Oral probiotics prevent necrotizing enterocolitis in very low birth weight neonates. J Pediatr. 2005;147:192-6.

41. Lin $\mathrm{H}-\mathrm{C}$, Su B-H, Chen A-C, Lin T-W, Tsai C-H, Yeh T-F, et al. Oral probiotics reduce the incidence and severity of necrotizing enterocolitis in very low birth weight infants. Pediatrics. 2005;115:1-4.

42. Dani C, Biadaioli R, Bertini G, Martelli E, Rubaltelli FF. Probiotics feeding in prevention of urinary tract infection, bacterial sepsis and necrotizing enterocolitis in preterm infants. Biol Neonate. 2002;82:103-8. 
43. Mihatsch WA, Vossbeck S, Eikmanns B, Hoegel J, Pohlandt F. Effect of Bifidobacterium lactis on the incidence of nosocomial infections in very-low-birth-weight infants: a randomized controlled trial. Neonatology. 2010:98:156-63.

44. Dilli D, Aydin B, Fettah N, Özyazıcı E, Beken S, Zenciroğlu A, et al. The ProPre-Save study: effects of probiotics and prebiotics alone or combined on necrotizing enterocolitis in very low birth weight infants $€$. J Pediatr. 2015. In press

45. Patole S, Keil AD, Chang A, Nathan E, Doherty D, Simmer K, et al. Effect of Bifidobacterium breve M-16 V supplementation on fecal bifidobacteria in preterm neonates-a randomised double blind placebo controlled trial. PLoS One. 2014;9:e89511.

46. Totsu S, Yamasaki C, Terahara M, Uchiyama A, Kusuda S. Bifidobacterium and enteral feeding in preterm infants: Cluster-randomized trial. Pediatr Int. 2014:56:714-9.

47. Sehgal A, Mak W, Dunn M, Kelly E, Whyte $H$, McCrindle B, et al. Haemodynamic changes after delivery room surfactant administration to very low birth weight infants. Arch Dis Child Fetal Neonatal Ed. 2010;95:F345-51

48. Saengtawesin V, Tangpolkaiwalsak R, Kanjanapattankul W. Effect of oral probiotics supplementation in the prevention of necrotizing enterocolitis among very low birth weight preterm infants. J Med Assoc Thai. 2014;97:S20-5.

49. Costalos C, Skouteri V, Gounaris A, Sevastiadou S, Triandafilidou A, Ekonomidou C, et al. Enteral feeding of premature infants with Saccharomyces boulardii. Early Hum Dev. 2003;74:89-96.

50. Rougé C, Piloquet $H$, Butel M-J, Berger B, Rochat F, Ferraris L, et al. Oral supplementation with probiotics in very-low-birth-weight preterm infants: a randomized, double-blind, placebo-controlled trial. Am J Clin Nutr. 2009:89:1828-35

51. Manzoni P, Mostert M, Leonessa ML, Priolo C, Farina D, Monetti C, et al. Oral supplementation with Lactobacillus casei subspecies rhamnosus prevents enteric colonization by Candida species in preterm neonates: a randomized study. Clin Infect Dis. 2006;42:1735-42.

52. Kitajima H, Sumida Y, Tanaka R, Yuki N, Takayama H, Fujimura M. Early administration of Bifidobacterium breve to preterm infants: randomised controlled trial. Arch Dis Child Fetal Neonatal Ed. 1997:76:F101-7.

53. Mohan R, Koebnick C, Schildt J, Schmidt S, Mueller M, Possner M, et al. Effects of Bifidobacterium lactis Bb12 supplementation on intestinal microbiota of preterm infants: a double-blind, placebo-controlled, randomized study. J Clin Microbiol. 2006;44:4025-31.

54. Li Y, Shimizu T, Hosaka A, Kaneko N, Ohtsuka Y, Yamashiro Y. Effects of bifidobacterium breve supplementation on intestinal flora of low birth weight infants. Pediatr Int. 2004:46:509-15.

55. Millar MR, Bacon C, Smith SL, Walker V, Hall MA. Enteral feeding of premature infants with Lactobacillus GG. Arch Dis Child. 1993;69:483-7.

56. Reuman PD, Duckworth DH, Smith KL, Kagan R, Bucciarelli RL, Ayoub EM Lack of effect of Lactobacillus on gastrointestinal bacterial colonization in premature infants. Pediatr Infect Dis. 1986;5:663-8.

57. Manzoni $P$, Rinaldi M, Cattani S, Pugni L, Romeo MG, Messner H. Bovine lactoferrin supplementation for prevention of late-onset sepsis in very low-birth-weight neonates. JAMA. 2009;302:1421-8.

58. Roy A, Chaudhuri J, Sarkar D, Ghosh P, Chakraborty S. Role of enteric supplementation of Probiotics on late-onset sepsis by Candida species in preterm low birth weight neonates: a randomized, double blind, placebocontrolled trial. N Am J Med Sci. 2014:6:50-7.

59. Alfaleh K, Anabrees J, Bassler D. Probiotics for prevention of necrotizing enterocolitis in preterm infants. Cochrane Database Syst Rev. 2011;3: CD005496. doi:10.1002/14651858.CD005496.pub3.

60. Drago L, De Vecchi E. Should Lactobacillus sporogenes and Bacillus coagulans have a future? J Chemother. 2009;21:371-7.

61. Guarner F, Malagelada J-R. Gut flora in health and disease. Lancet. 2003;361:512-9.

62. Luedtke SA, Yang JT, Wild HE. Probiotics and necrotizing enterocolitis: finding the missing pieces of the probiotic puzzle. J Pediatr Pharmacol Ther. 2012;17:308-28.

63. Berrington JE, Stewart CJ, Embleton ND, Cummings SP. Gut microbiota in preterm infants: assessment and relevance to health and disease. Arch Dis Child Fetal Neonatal Ed. 2013;98:F286-90.

64. Boyd CA, Quigley MA, Brocklehurst P. Donor breast milk versus infant formula for preterm infants: systematic review and meta-analysis. Arch Dis Child Fetal Neonatal Ed. 2007:92:F169-75.
65. Penders J, Thijs C, Vink C, Stelma FF, Snijders B, Kummeling I, et al. Factors influencing the composition of the intestinal microbiota in early infancy. Pediatrics. 2006;118:511-21

66. Beattie LM, Hansen R, Barclay AR. Probiotics for preterm infants: confounding features warrant caution. Pediatrics. 2010;126:e742-3. author reply e743-5.

67. Kelsen JR, Wu GD. The gut microbiota, environment and diseases of modern society. Gut Microbes. 2012;3:374-82.

\section{Submit your next manuscript to BioMed Central and take full advantage of:}

- Convenient online submission

- Thorough peer review

- No space constraints or color figure charges

- Immediate publication on acceptance

- Inclusion in PubMed, CAS, Scopus and Google Scholar

- Research which is freely available for redistribution 